\title{
Theoretical Vibration Analysis Regarding Excitation due to Elliptical Shaft Journals in Sleeve Bearings of Electrical Motors
}

\author{
Ulrich Werner \\ Research and Development Department, Siemens AG, I DT LD P R\&D, 90441 Nuremberg, Germany \\ Correspondence should be addressed to Ulrich Werner, werner.ulrich@siemens.com
}

Received 23 September 2011; Accepted 25 November 2011

Academic Editor: Jerzy Sawicki

Copyright () 2012 Ulrich Werner. This is an open access article distributed under the Creative Commons Attribution License, which permits unrestricted use, distribution, and reproduction in any medium, provided the original work is properly cited.

This paper shows a theoretical vibration analysis regarding excitation due to elliptical shaft journals in sleeve bearings of electrical motors, based on a simplified rotordynamic model. It is shown that elliptical shaft journals lead to kinematic constraints regarding the movement of the shaft journals on the oil film of the sleeve bearings and therefore to an excitation of the rotordynamic system. The solution of the linear differential equation system leads to the mathematical description of the movement of the rotor mass, the shaft journals, and the sleeve bearing housings. Additionally the relative movements between the shaft journals and the bearing housings are deduced, as well as the bearing housing vibration velocities. The presented simplified rotordynamic model can also be applied to rotating machines, other than electrical machines. In this case, only the electromagnetic spring value $c_{m}$ has to be put to zero.

\section{Introduction}

Many different kinds of excitation exist in rotating machinery-for example, mechanical unbalance and misalignment of the coupling-which may cause vibrations [1-6]. Besides these typical excitations also specific excitations associated with the type of the rotating machine occur. This paper focuses on vibrations of electrical machines; therefore also electromagnetic forces have to be considered, which may cause vibrations. These electromagnetic forces mainly occur if an eccentricity of the air gap in the electrical machine exists. The eccentricity can be divided into static eccentricity-that means the smallest air gap remains at constant position-and dynamic eccentricity, which means that the smallest air gap changes its position by the rotation of the rotor. Static eccentricity is, for example, caused by production tolerances regarding concentricity and fitting tolerance between stator housing, end shields, bearing housing, and so forth. Dynamic eccentricity is caused if the rotor is bent or if the rotor core is eccentrically positioned on the rotor shaft due to, for example, tolerances in the punching process of the rotor sheets. In this case a rotating magnetic force occurs, which is called UMP (unbalanced magnetic pull), which has been investigated in many publications as in Dorrell
[7], Smith and Dorrell [8], Schuisky [9], Holopainen [10], Arkkio et al. [11], Belmans et al. [12], Stoll [13], and Werner $[14,15]$.

Most of the vibrations in rotating machinery occur with the frequency of the rotor rotation $(f=1 x)[1-5]$, for example, mechanical unbalance or UMP in electrical machines. Due to misalignment of the coupling between the rotating machines also multiple frequencies of the rotor rotation occur in the vibration spectrum $(f=1 x, 2 x, 3 x, 4 x, \ldots)$. Due to instability - for example, caused in fast rotating machines, where the rotors are supported by sleeve bearings-also subsynchronous vibrations $(f<1 x)$, know as self-excited vibrations, may occur [1-6].

In this paper a special kind of excitation is investigated. When machining the rotor-turning and grinding-the shaft journals may be subject to a marginal form deviation, so that they are no longer absolutely cylindrical. In some cases the shaft journals can get an elliptical shape instead of circular shape (Figure 1). This form deviation $\Delta$ of the circular shape of the shaft journals is usually very small, about $0.0005-0.002 \%$ referred to the diameter of the shaft journal. In standards and specifications the so-called run out of the shaft journals is limited by, for example, the standard IEC 60034-14 [16] and the standard API 541 [17]. But due to 


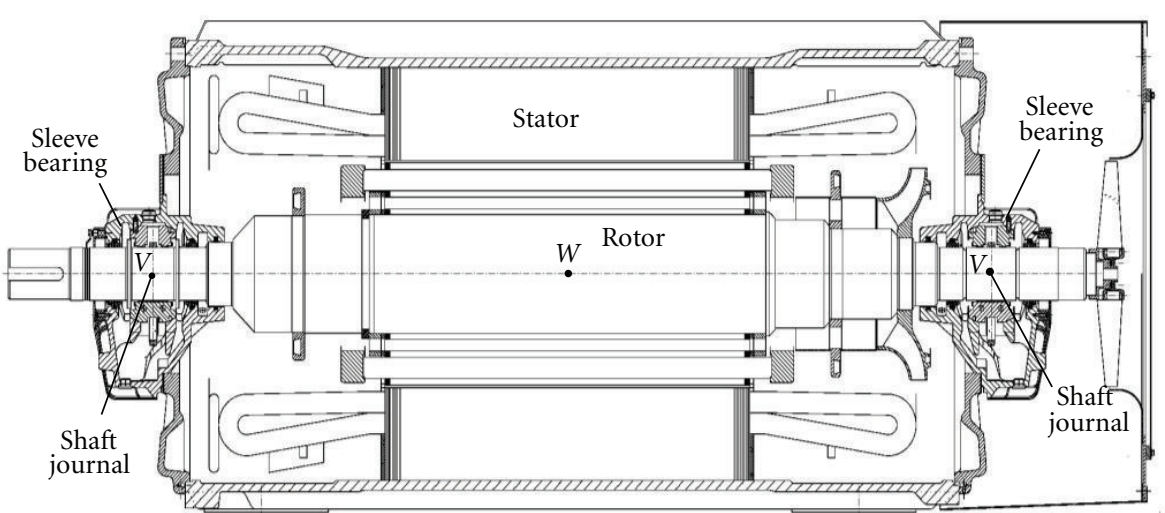

(a)

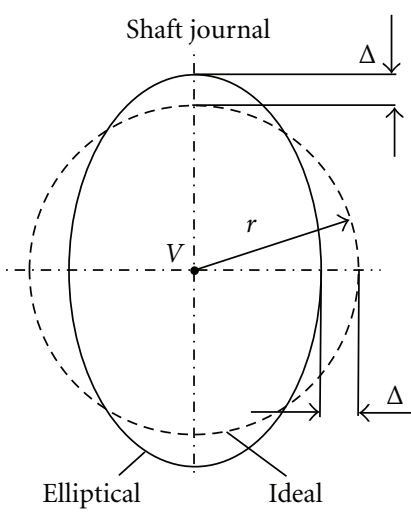

(b)

FIGURE 1: (a) Longitudinal section of a 2-pole induction motor with sleeve bearings. (b) Excitation due to elliptical shaft journals.

this form deviation the centre of the shaft journal $s$ changes its position as the rotor rotates. As a result of this movement of the shaft journal point $V$ dynamic forces will occur in the oil film of the sleeve bearing and the centre of the rotor mass $W$ will also move (Figure 1). The dynamic displacement of the centre of the rotor $W$ causes an acceleration force, acting at the rotor centre $W$. In addition, the air gap of the electrical machine will change due to the displacement of the rotor centre $W$ and electromagnetic forces will occur.

The aim of this paper is to show a theoretical vibration analysis regarding excitation due to elliptical shaft journals in sleeve bearings of electrical motors, based on a simplified rotordynamic model. With this simplified model the relative shaft displacements between the sleeve bearing housings and the shaft journals can be shown as well as the vibration velocity of the sleeve bearing housings.

\section{Hydrodynamic Oil Film Forces}

The characteristic of a hydrodynamic oil film is well known and described in, for example, Friswell et al. [1], Maurice and Adams [2], Vance et al. [3], Rao [4], Gasch et al. [5], Kliem et al. [6] and in Reynolds [18], Gnanadoss and Osborne [19], Glienicke [20], Lund and Thomsen [21], and Tondl [22]. Due to the rotor rotation - the rotor rotates with the rotary angular frequency $\Omega-$ a hydrodynamic oil film between the shaft journal and the bearing shell is created (Figure 2(a)). By changing the rotor speed, the shaft journal point $V$ moves on a curve, which is known as the Gümbel curve (Gasch et al. [5]), until the final rotor speed is reached. This position is the static rest position of the shaft journal point $V$, which can be described by the static eccentricity $e$ and the angle $\beta$ (Figure 2(a)). In this analysis, the shaft journal has an ideal circular shape.

The static rest position of the shaft journal point $V$ depends on following parameters, which is well known:

(i) rotor angular frequency $\Omega$,

(ii) static bearing forces, caused by for example, the rotor weight and static magnetic pull, (iii) shape of the bearing shell, for example, cylindrical, 2-lobe, 4-lobe, and so forth,

(iv) bearing clearance,

(v) oil grade,

(vi) oil film temperature.

If no additional forces act on the shaft journal, the shaft journal point $V$ will remain at the static rest position. If additional forces $F_{z}$ and $F_{y}$ act on the shaft journal point $V$ a relative displacement between the centre of the bearing shell $B$-which is assumed here to be also the centre of the bearing housing —and the shaft journal point $V$ will occur, which will lead to additional oil film forces. This coherence is well known and can be mathematically described as follows:

$$
\underbrace{\left[\begin{array}{c}
F_{z} \\
F_{y}
\end{array}\right]}_{\mathbf{F}}=\underbrace{\left[\begin{array}{c}
c_{z z} c_{z y} \\
c_{y z} c_{y y}
\end{array}\right]}_{\mathbf{C}_{v}} \cdot \underbrace{\left[\begin{array}{c}
z_{v}-z_{b} \\
y_{v}-y_{b}
\end{array}\right]}_{\mathbf{q}_{\mathrm{rel}}}+\underbrace{\left[\begin{array}{c}
d_{z z} d_{z y} \\
d_{y z} d_{y y}
\end{array}\right]}_{\mathbf{D}_{v}} \cdot \underbrace{\left[\begin{array}{c}
\dot{z}_{v}-\dot{z}_{b} \\
\dot{y}_{v}-\dot{y}_{b}
\end{array}\right]}_{\mathbf{q}_{\mathrm{rel}}} .
$$

The oil film stiffness matrix $\mathbf{C}_{v}$ contains the oil film stiffness coefficients $\left(c_{y y}, c_{y z}, c_{z y}, c_{z z}\right)$ and the oil film damping matrix $\mathbf{D}_{v}$ contains the oil film damping coefficients $\left(d_{y y}\right.$, $d_{y z}, d_{z y}, d_{z z}$ ), which can be derived by solving the ReynoldsDifferential Equations [18, 19]. Vector $\mathbf{q}_{\text {rel }}$ describes the relative movement between the shaft journal point $V$ and the bearing centre point $B$ and vector $\dot{\mathbf{q}}_{\text {rel }}$ describes the relative velocity between the shaft journal point $V$ and the bearing centre point $B$. But also forced displacements between shaft journal and bearing shell, for example, caused by elliptical shaft journals, will lead to additional oil film forces, which is the focus of the paper.

The relative displacement between the shaft journal point $V$ and its static rest position is very important for the evaluation of the vibrations. The static rest position of the shaft journal in the bearing shell is linked to the centre of the bearing shell $B$, described by the eccentricity $e$ and the angle $\beta$ (Figure 2(a)). Therefore the static rest position of the shaft journal is here defined to be static relative to the centre of the 


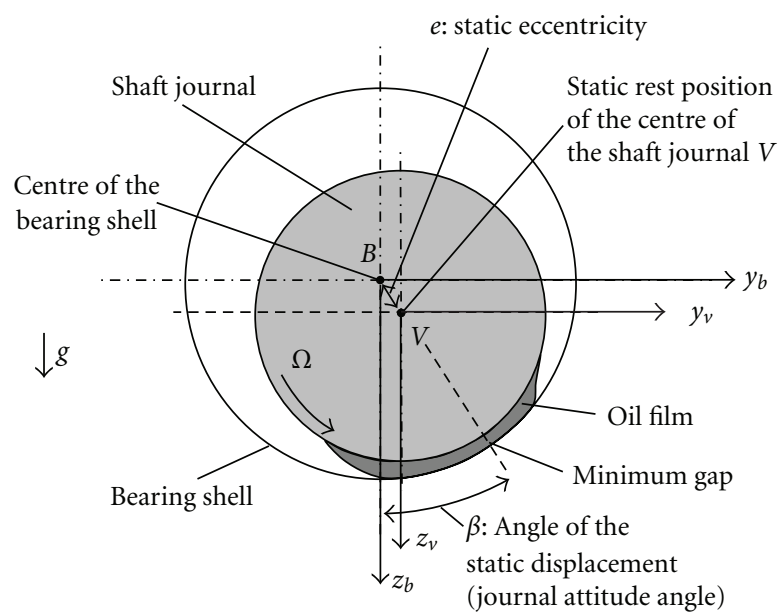

(a)

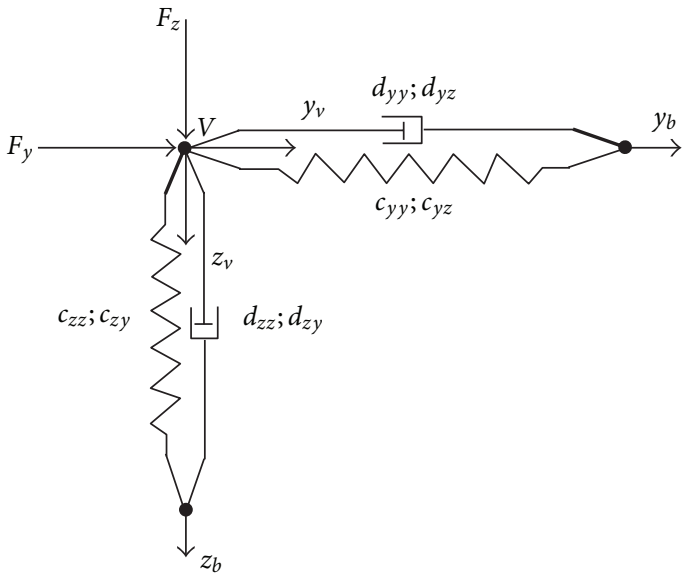

(b)

Figure 2: (a) Static rest position of the shaft journal point $V$ in a hydrodynamic sleeve bearing. (b) Additional forces $F_{z}$ and $F_{y}$ at the static rest position causing relative displacements between the centre of the bearing shell $B$ and the shaft journal $V$.

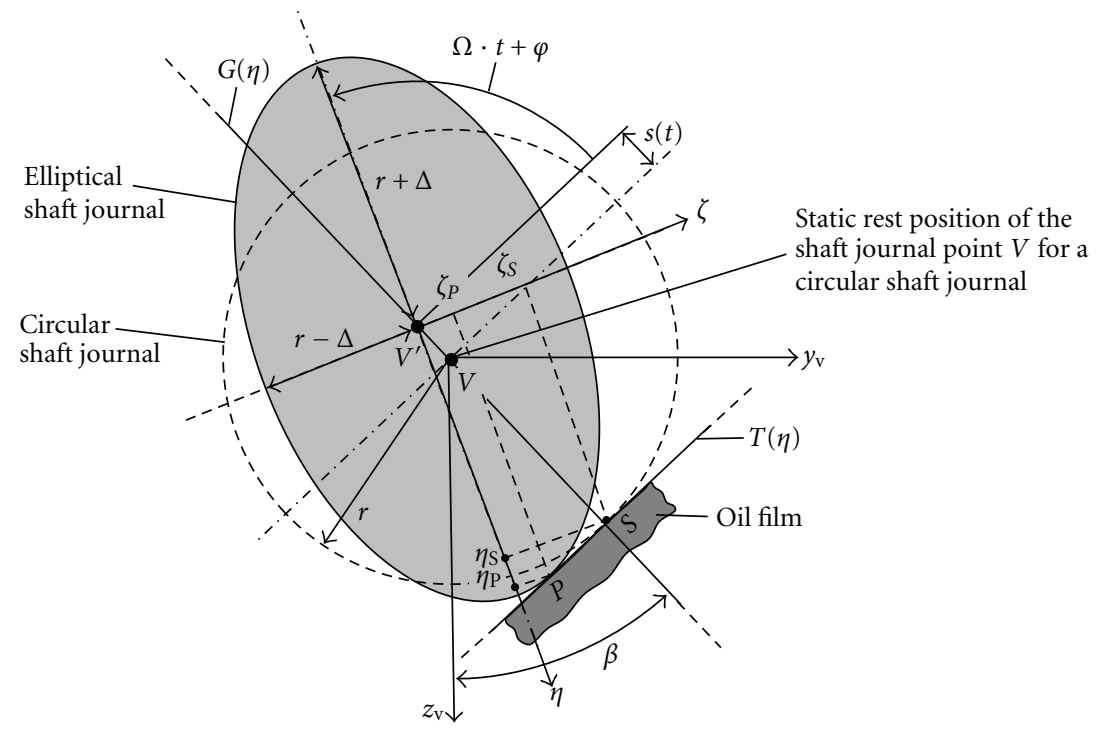

Figure 3: Forced displacement $s(t)$ of the shaft journal point $V$ on an oil film due to the rotation of an elliptical shaft journal.

bearing shell $B$. So if a displacement of the bearing housing itself occurs, also the static rest position of the shaft journal is moved. Therefore the relative displacement between the shaft journal point $V$ and the bearing centre point $B$ is chosen here to evaluate the vibrations.

\section{Forced Displacement of the Shaft Journal}

In this section the forced displacement $s(t)$ of the shaft journal point $V$ on the oil film of the sleeve bearing is mathematically derived while the rotor rotates with the rotary angular frequency $\Omega$. In this analysis the forced displacement $s(t)$ is only caused by the elliptical shape of the shaft journal. Further, only a normal displacement regarding the oil film, which means in direction of the eccentricity $e$ with the angle $\beta$, is assumed (Figure 3). The ellipse function of the shaft journal can be described in the coordinate system $(\eta ; \zeta)$ by

$$
\frac{\eta^{2}}{(r+\Delta)^{2}}+\frac{\zeta^{2}}{(r-\Delta)^{2}}=1
$$

The tangent function $T(\eta)$ through the point $P$ is described by

$$
T(\eta)=-\frac{(r-\Delta)^{2}}{(r+\Delta)^{2}} \cdot \frac{\eta_{P}}{\zeta_{P}} \cdot \eta+\frac{(r-\Delta)^{2}}{\zeta_{P}} .
$$


The function $G(\eta)$ is described by

$$
G(\eta)=\eta \cdot \tan \left[\frac{\pi}{2}-(\Omega \cdot t+\varphi)\right]
$$

According to Figure 3 the tangent function $T(\eta)$ has to be normal to the function $G(\eta)$. With this boundary condition follows:

$$
\tan \left[\frac{\pi}{2}-(\Omega \cdot t+\varphi)\right]=\frac{(r+\Delta)^{2}}{(r-\Delta)^{2}} \cdot \frac{\zeta_{P}}{\eta_{P}} .
$$

The second boundary condition is that the tangent point $P$ described by the coordinates $\eta_{P}$ and $\zeta_{P}$-lies on the ellipse, which leads to the condition

$$
\frac{\eta_{P}^{2}}{(r+\Delta)^{2}}+\frac{\zeta_{P}^{2}}{(r-\Delta)^{2}}=1
$$

With the boundary conditions (5) and (6) the coordinates $\eta_{P}$ and $\zeta_{P}$ of the tangent point $P$ can be calculated:

$$
\begin{gathered}
\eta_{P}=\frac{(r+\Delta)^{2}}{\sqrt{(r-\Delta)^{2} \cdot \tan ^{2}[\pi / 2-(\Omega \cdot t+\varphi)]+(r+\Delta)^{2}}}, \\
\zeta_{P}=\frac{(r-\Delta)^{2} \cdot \tan [\pi / 2-(\Omega \cdot t+\varphi)]}{\sqrt{(r-\Delta)^{2} \cdot \tan ^{2}[\pi / 2-(\Omega \cdot t+\varphi)]+(r+\Delta)^{2}}} .
\end{gathered}
$$

The tangent function $T(\eta)$ (3) is now completely described with the coordinates $\eta_{P}$ and $\zeta_{P}$. The intersection point $S$ between the function $G(\eta)$ and the tangent function $T(\eta)$ can be calculated, and the coordinate $\eta_{S}$ can be derived:

$\eta_{S}=\frac{(r-\Delta)^{2}}{\left((r-\Delta)^{2} /(r+\Delta)^{2}\right) \cdot \eta_{P}+\zeta_{P} \cdot \tan [\pi / 2-(\Omega \cdot t+\varphi)]}$.

Now the segment coordinate $\overline{S V^{\prime}}$ can be derived:

$$
\begin{aligned}
\overline{S V^{\prime}} & =\frac{1}{\cos [(\pi / 2)-(\Omega \cdot t+\varphi)]} \cdot \eta_{\mathrm{S}} \\
& =\sqrt{(r-\Delta)^{2} \cdot \cos ^{2}(\Omega \cdot t+\varphi)+(r+\Delta)^{2} \cdot \sin ^{2}(\Omega \cdot t+\varphi)} .
\end{aligned}
$$

The dynamic displacement $s(t)$ can be calculated with the segment $\overline{S V^{\prime}}$ :

$$
s(t)=\sqrt{(r-\Delta)^{2} \cdot \cos ^{2}(\Omega \cdot t+\varphi)+(r+\Delta)^{2} \cdot \sin ^{2}(\Omega \cdot t+\varphi)}-r .
$$

Using the following correlations:

$$
\begin{gathered}
\cos ^{2}(\Omega \cdot t+\varphi)=\frac{1}{2}[\cos (2 \Omega \cdot t+2 \varphi)+1], \\
\sin ^{2}(\Omega \cdot t+\varphi)=-\frac{1}{2}[\cos (2 \Omega \cdot t+2 \varphi)-1],
\end{gathered}
$$

the displacement $s(t)$ becomes:

$$
\begin{aligned}
& s(t) \\
& =\sqrt{[r-\Delta \cdot \cos (2 \Omega \cdot t+2 \varphi)]^{2}-\Delta^{2} \cdot \cos ^{2}(2 \Omega \cdot t+2 \varphi)+\Delta^{2}}-r .
\end{aligned}
$$

With the boundary condition $(\Delta \ll r)$ the terms with $\Delta^{2}$ can be neglected and the displacement $s(t)$ of the shaft journal point $V$ can be described by

$$
s(t) \approx-\Delta \cdot \cos (2 \Omega \cdot t+2 \varphi) .
$$

Therefore the shaft journal point $V$ lifts and lowers two times per rotor rotation $(2 x)$.

\section{Rotordynamic Model}

The rotor dynamic model is a simplified rotor model based on the well-known JEFFCOTT rotor, which is described in many publication as, for example, in Friswell et al. [1], Maurice and Adams [2], Vance et al. [3], Rao [4], and Gasch et al. [5]. For electrical motors the model is enhanced here by considering electromagnetic coupling between the rotor and the stator. Additionally a nonsymmetric position of the rotor mass $\left(l_{1} \neq l_{2}\right)$ and different bearings (1) and (2), with the different oil film stiffness and damping coefficients, and different stiffness of the bearing housings and of the end shields on both sides, is considered in this model. However, the most important feature of this simplified rotordynamic model is the excitation due to the elliptical shaft journals (Figure 4).

The total rotor mass $m$ is concentrated as a lumped mass on an elastic, massless shaft with the rotor stiffness $c$. Moments of inertia are not taken into consideration. The rotor rotates with the angular frequency $\Omega$. The point $W$, which is positioned at the axial middle of the rotor core, describes the movement of the shaft centre point of the rotor. The shaft journal points $V_{1}$ and $V_{2}$ describe the movement of the shaft journals in the sleeve bearings and the bearing housing centre points $B_{1}$ and $B_{2}$ the movement of the centre of the bearing housings.

The oil film stiffness and damping of the sleeve bearings are considered by the oil film stiffness matrices $\mathbf{C}_{v 1}$ and $\mathbf{C}_{v 2}$ the oil film damping matrices $\mathbf{D}_{v 1}$ and $\mathbf{D}_{v 2}$, which include the oil film stiffness coefficients and the oil film damping coefficients of each sleeve bearings. The vertical stiffness $c_{b z 1}$ and $c_{b z 2}$ and the horizontal stiffness $c_{b y 1}$ and $c_{b y 2}$ - which are included into the stiffness matrices $\mathbf{C}_{b 1}$ and $\mathbf{C}_{b 2}$ - describe a series connection in vertical and horizontal direction of the stiffness of the sleeve bearing housing, the end shield, and the stator housing in the area of the end shield. Usually the stiffness of the stator housing in the area of the end shields is so high that it can be assumed to be rigid when compared to the stiffness of the bearing housing and end shield. The foundation is assumed to be rigid in this model, simulating a massive foundation. Therefore no foundation stiffness has to be considered. Damping of the bearing housings and end shields is not considered because of the usually low damping values of cast iron. 


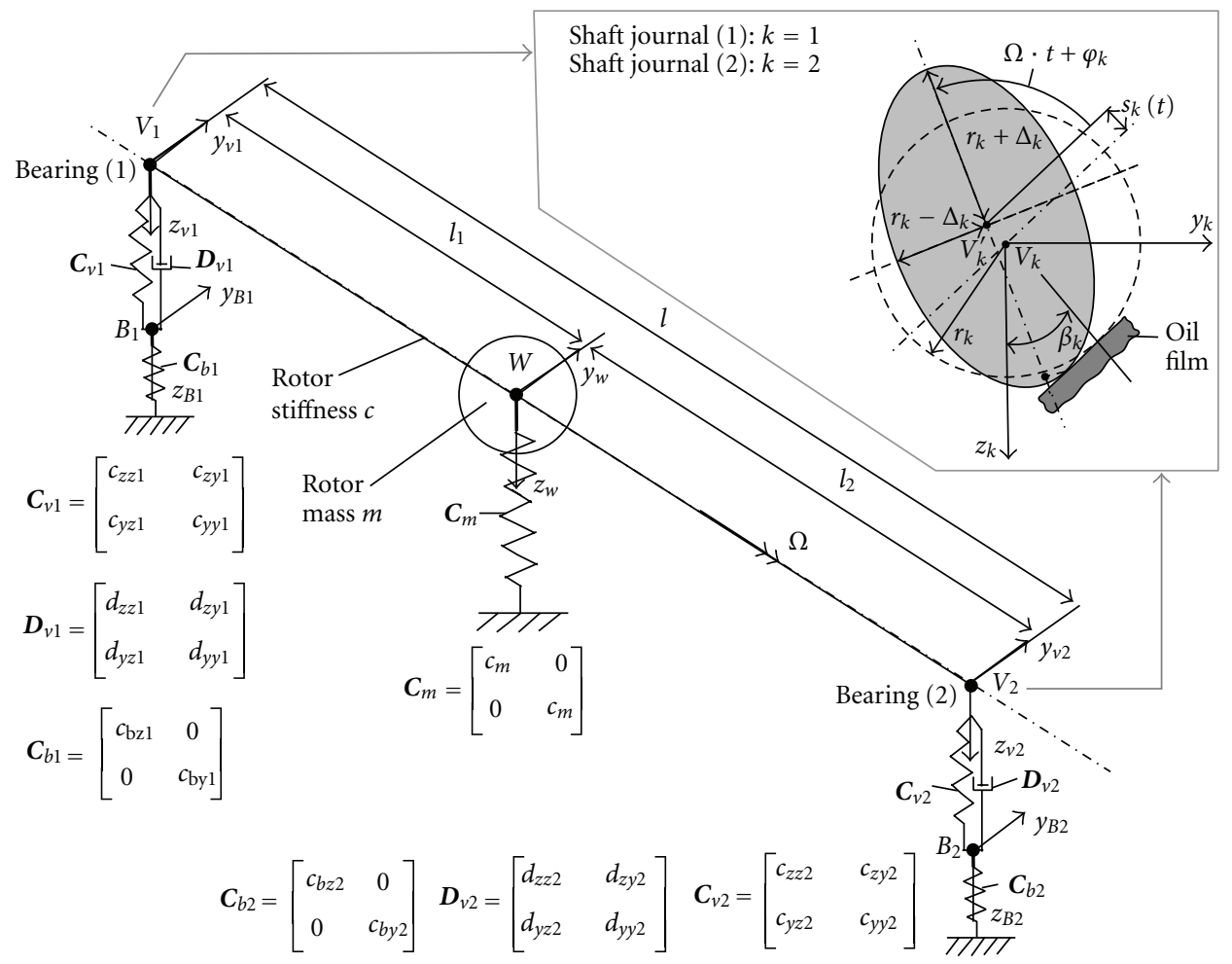

FIgURE 4: Rotordynamic model.

For electrical machines, there is an electromagnetic coupling between the rotor and the stator, which can be described by the magnetic spring matrix $\mathbf{C}_{m}$, which contains the magnetic spring constant $c_{m}$. The magnetic spring constant $c_{m}$ has a negative reaction, which is shown in Dorrell [7], Smith and Dorrell [8], Schuisky [9], Holopainen [10], Arkkio et al. [11], Belmans et al. [12], Stoll [13], and Werner [14, 15]. This means that a radial movement between the rotor and stator creates an electromagnetic force that tries to magnify the movement. In this paper, the magnetic spring constant is defined to be positive and acts in the direction of the magnetic forces. Electromagnetic field damping is not considered in the simplified model.

The excitation in this rotordynamic model is only caused by elliptical shaft journals. Other excitations like mechanical unbalance, unbalanced magnetic pull, and so forth are not considered in this analysis, but they can be superposed. Therefore, the excitation is only caused by the forced displacement of each elliptical shaft journal on the oil film in each sleeve bearing. According to (14) the forced displacement $s_{1}(t)$ and $s_{2}(t)$ of the shaft journal centre point $V_{1}$ and $V_{2}$ can be described by:

$$
s_{k}(t)=-\Delta_{k} \cdot \cos \left(2 \Omega \cdot t+2 \varphi_{\mathrm{k}}\right)
$$

with: $k=1$ for bearing (1); $k=2$ for bearing (2).

The form deviation $\Delta_{k}$ can be different for each shaft journal, as well as the angularity $\varphi_{k}$. This is the main reason, why each bearing — bearing (1) and bearing (2)—has to be modeled separately.

In the rotordynamic model, there are five fixed coordinate systems. The displacement of the shaft centre point $W$ is described in the coordinate system $\left(z_{w} ; y_{w}\right)$. The displacements of the shaft journal points $V_{1}$ and $V_{2}$ are described in the coordinate systems $\left(z_{V 1} ; y_{V 1}\right)$ and $\left(z_{V 2} ; y_{V 2}\right)$, and the displacements of the bearing housing centre points $B_{1}$ and $B_{2}$ in the coordinate systems $\left(z_{B 1} ; y_{B 1}\right)$ and $\left(z_{B 2} ; y_{B 2}\right)$. The coordinate systems $\left(z_{V 1} ; y_{V 1}\right)$ and $\left(z_{B 1} ; y_{B 1}\right)$ as well as the coordinate systems $\left(z_{V 2} ; y_{V 2}\right)$ and $\left(z_{B 2} ; y_{B 2}\right)$ are shown with a clear offset to illustrate the spring/damper connections between $V_{1}$ and $B_{1}$ and between $V_{2}$ and $B_{2}$. In effect, only the static sleeve bearing eccentricity $e$ determines the offset (Figure 2(a)).

\section{Mathematical Description of the Rotordynamic Model}

5.1. Differential Equation System. To derive the differential equation system of the rotordynamic model it is necessary to split up the vibration model into subsystems and to describe the forces that act in each subsystem (Figure 5):

(a) subsystem (Figure 5(a)) for the forces acting at the rotor mass $m$ at the shaft centre point $W$;

(b) subsystem (Figure 5(b)) for the forces acting at the shaft journal points $V_{1}$ and $V_{2}(k=1,2)$;

(c) subsystems (Figure 5(c)) for the forces acting at the bearing housing points $B_{1}$ and $B_{2}(k=1,2)$. 


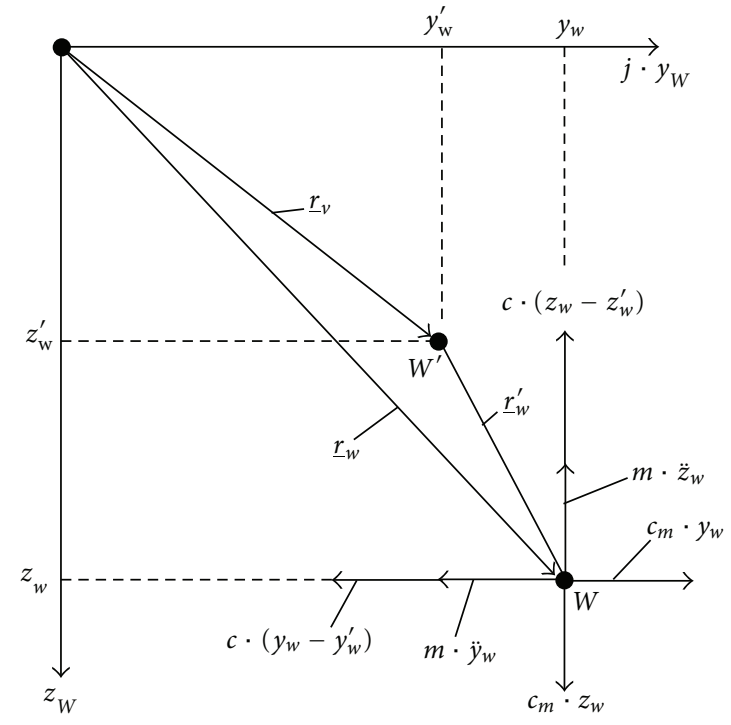

(a)

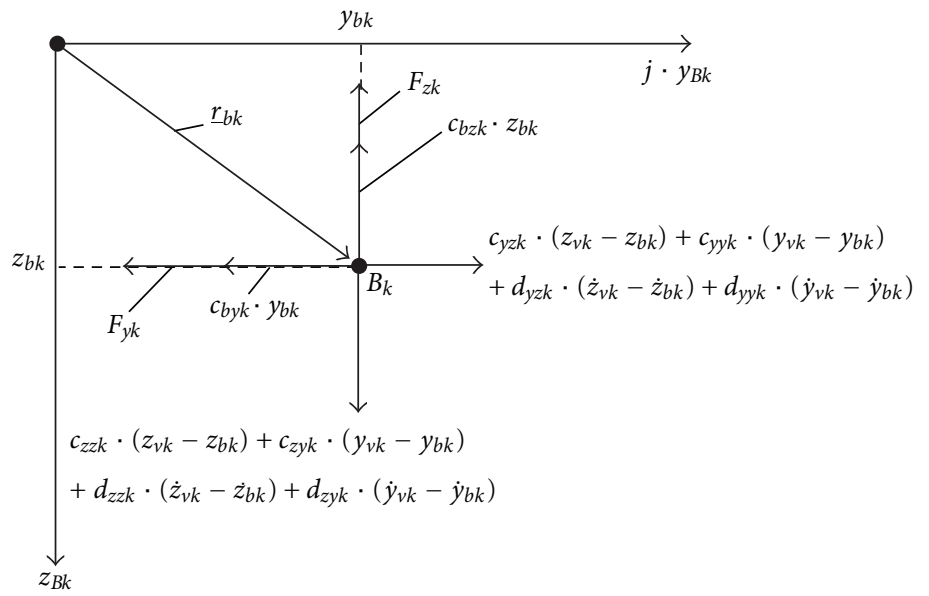

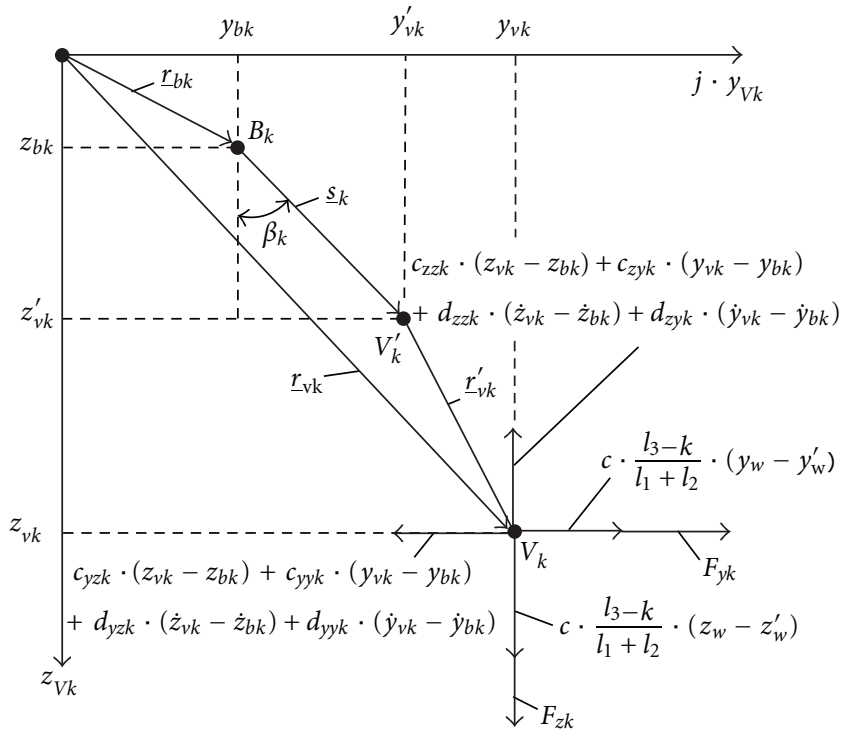

(b)

(c)

FIgURE 5: Vibration model, split up into subsystems: (a) rotor mass system, (b) shaft journal system, (c) bearing housing system.

Additionally, complex coordinate systems are introduced for a subsequent mathematical analysis.

5.1.1. Rotor Mass System. The equilibrium of forces at the centre of the rotor mass $m$ at the shaft centre point $W$ can be described by the following equations:

$$
\begin{aligned}
& m \cdot \ddot{z}_{w}+c \cdot\left(z_{w}-z_{w}^{\prime}\right)-c_{m} \cdot z_{w}=0, \\
& m \cdot \ddot{y}_{w}+c \cdot\left(y_{w}-y_{w}^{\prime}\right)-c_{m} \cdot y_{w}=0,
\end{aligned}
$$

with the kinematic constraints

$$
\begin{aligned}
& z_{w}^{\prime}=\sum_{k=1}^{2} \frac{l_{3-k}}{l} \cdot z_{v k}, \\
& y_{w}^{\prime}=\sum_{k=1}^{2} \frac{l_{3-k}}{l} \cdot y_{v k} .
\end{aligned}
$$

5.1.2. Journal Shaft System ( $k=1$ for Bearing (1) and $k=2$ for Bearing (2)). The equilibrium of forces at the shaft journal points $V_{1}$ and $V_{2}$ are described by

$$
\begin{aligned}
& c_{z z k} \cdot\left(z_{v k}-z_{b k}\right)+c_{z y k} \cdot\left(y_{v k}-y_{b k}\right) \\
&+d_{z z k} \cdot\left(\dot{z}_{v k}-\dot{z}_{b k}\right)+d_{z y k} \cdot\left(\dot{y}_{v k}-\dot{y}_{b k}\right) \\
&-c \cdot \frac{l_{3-k}}{l} \cdot\left[z_{w}-\frac{l_{2}}{l} \cdot z_{v 1}-\frac{l_{1}}{l} \cdot z_{v 2}\right]-F_{z k}=0, \\
& c_{y z k} \cdot\left(z_{v k}-z_{b k}\right)+c_{y y k} \cdot\left(y_{v k}-y_{b k}\right) \\
& \quad+d_{y z k} \cdot\left(\dot{z}_{v k}-\dot{z}_{b k}\right)+d_{y y k} \cdot\left(\dot{y}_{v k}-\dot{y}_{b k}\right) \\
&-c \cdot \frac{l_{3-k}}{l} \cdot\left[y_{w}-\frac{l_{2}}{l} \cdot y_{v 1}-\frac{l_{1}}{l} \cdot y_{v 2}\right]-F_{y k}=0 .
\end{aligned}
$$

The oil film forces $F_{z k}$ and $F_{y k}$, which are caused by the forced displacements $s_{k}(t)$ and which act at the shaft journal points 
$V_{k}$ and in opposite direction at the bearing housing points $B_{k}$, are described by

$$
\begin{aligned}
F_{z k}= & c_{z z k} \cdot \cos \beta_{k} \cdot s_{k}(t)+c_{z y k} \cdot \sin \beta_{k} \cdot s_{k}(t) \\
& +d_{z z k} \cdot \cos \beta_{k} \cdot \dot{s}_{k}(t)+d_{z y k} \cdot \sin \beta_{k} \cdot \dot{s}_{k}(t), \\
F_{y k}= & c_{y z k} \cdot \cos \beta_{k} \cdot s_{k}(t)+c_{y y k} \cdot \sin \beta_{k} \cdot s_{k}(t) \\
& +d_{y z k} \cdot \cos \beta_{k} \cdot \dot{s}_{k}(t)+d_{y y k} \cdot \sin \beta_{k} \cdot \dot{s}_{k}(t) .
\end{aligned}
$$

With (15) the oil film forces become

$$
\begin{aligned}
F_{z k}= & \left(-c_{z z k} \cdot \Delta_{k} \cdot \cos \beta_{k}-c_{z y k} \cdot \Delta_{k} \cdot \sin \beta_{k}\right) \\
& \cdot \cos \left(2 \Omega \cdot t+2 \varphi_{k}\right) \\
& +\left(d_{z z k} \cdot \Delta_{k} \cdot 2 \Omega \cdot \cos \beta_{k}+d_{z y k} \cdot \Delta_{k} \cdot 2 \Omega \cdot \sin \beta_{k}\right) \\
& \cdot \sin \left(2 \Omega \cdot t+2 \varphi_{k}\right), \\
F_{y k}= & \left(-c_{y z k} \cdot \Delta_{k} \cdot \cos \beta_{k}-c_{y y k} \cdot \Delta_{k} \cdot \sin \beta_{k}\right) \\
& \cdot \cos \left(2 \Omega \cdot t+2 \varphi_{k}\right) \\
& +\left(d_{y z k} \cdot \Delta_{k} \cdot 2 \Omega \cdot \cos \beta_{k}+d_{y y k} \cdot \Delta_{k} \cdot 2 \Omega \cdot \sin \beta_{k}\right) \\
& \cdot \sin \left(2 \Omega \cdot t+2 \varphi_{k}\right) .
\end{aligned}
$$

5.1.3. Bearing Housing System ( $k=1$ for Bearing (1) and $k=$ 2 for Bearing (2)). The equilibrium of forces at the bearing housing points $B_{1}$ and $B_{2}$ is described by

$$
\begin{aligned}
& -c_{z z k} \cdot\left(z_{v k}-z_{b k}\right)-c_{z y k} \cdot\left(y_{v k}-y_{b k}\right) \\
& \quad-d_{z z k} \cdot\left(\dot{z}_{v k}-\dot{z}_{b k}\right)-d_{z y k} \cdot\left(\dot{y}_{v k}-\dot{y}_{b k}\right) \\
& \quad+c_{b z k} \cdot z_{b k}+F_{z k}=0, \\
& -c_{y z k} \cdot\left(z_{v k}-z_{b k}\right)-c_{y y k} \cdot\left(y_{v k}-y_{b k}\right)
\end{aligned}
$$

$$
\begin{aligned}
& -d_{y z k} \cdot\left(\dot{z}_{v k}-\dot{z}_{b k}\right)-d_{y y k} \cdot\left(\dot{y}_{v k}-\dot{y}_{b k}\right) \\
& +c_{b y k} \cdot y_{b k}+F_{y k}=0
\end{aligned}
$$

with the oil film forces (21).

Based on the equilibrium of forces, the differential equation system, described by the mass matrix $\mathbf{M}$, the damping matrix $\mathbf{D}$, the stiffness matrix $\mathbf{C}$, coordinate vector $\mathbf{q}$, and excitation vector $\mathbf{f}$, can be derived:

$$
\mathbf{M} \cdot \ddot{\mathbf{q}}+\mathbf{D} \cdot \dot{\mathbf{q}}+\mathbf{C} \cdot \mathbf{q}=\mathbf{f} .
$$

The differential equation system is a differential equation of second order. It is linear and inhomogeneous. The matrices are quadratic and have the shape $(m, n)$ with the dimension $m=n=10$ and the vectors are column vectors with the dimension $n=10$. by

Mass matrix $\mathbf{M}$ and the coordinate vector $\mathbf{q}$ are described

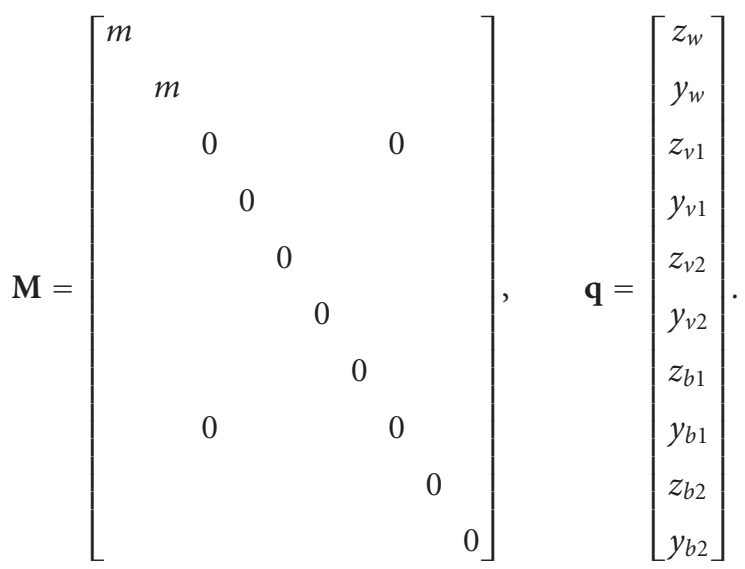

$$
\mathbf{D}=\left[\begin{array}{cccccccccc}
0 & 0 & 0 & 0 & 0 & 0 & 0 & 0 & 0 & 0 \\
0 & 0 & 0 & 0 & 0 & 0 & 0 & 0 & 0 & 0 \\
0 & 0 & d_{z z 1} & d_{z y 1} & 0 & 0 & -d_{z z 1} & -d_{z y 1} & 0 & 0 \\
0 & 0 & d_{y z 1} & d_{y y 1} & 0 & 0 & -d_{y z 1} & -d_{y y 1} & 0 & 0 \\
0 & 0 & 0 & 0 & d_{z z 2} & d_{z y 2} & 0 & 0 & -d_{z z 2} & -d_{z y 2} \\
0 & 0 & 0 & 0 & d_{y z 2} & d_{y y 2} & 0 & 0 & -d_{y z 2} & -d_{y y 2} \\
0 & 0 & -d_{z z 1} & -d_{z y 1} & 0 & 0 & d_{z z 1} & d_{z y 1} & 0 & 0 \\
0 & 0 & -d_{y z 1} & -d_{y y 1} & 0 & 0 & d_{y z 1} & d_{y y 1} & 0 & 0 \\
0 & 0 & 0 & 0 & -d_{z z 2} & -d_{z y 2} & 0 & 0 & d_{z z 2} & d_{z y 2} \\
0 & 0 & 0 & 0 & -d_{y z 2} & -d_{y y 2} & 0 & 0 & d_{y z 2} & d_{y y 2}
\end{array}\right] .
$$


The stiffness matrix $\mathbf{C}$ is described by

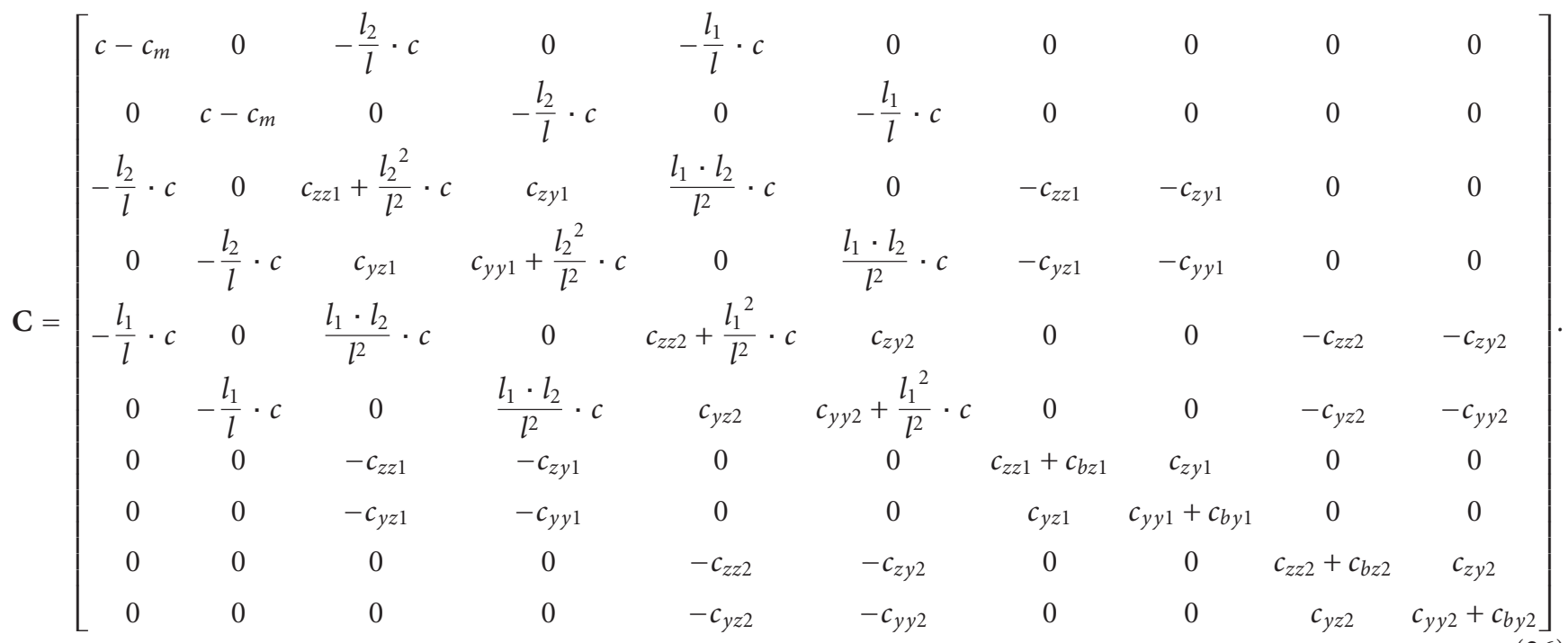

The excitation vector $\mathbf{f}$ is described by

$$
\widehat{\mathbf{f}}_{2, \sin }=\left[0 ; 0 ; 0 ; 0 ; \widehat{F}_{z 2, \sin } ; \widehat{F}_{y 2, \sin } ; 0 ; 0 ;-\widehat{F}_{z 2, \sin } ;-\widehat{F}_{y 2, \sin }\right]^{T}
$$

$$
\mathbf{f}=\left[0 ; 0 ; F_{z 1} ; F_{y 1} ; F_{z 2} ; F_{y 2} ;-F_{z 1} ;-F_{y 1} ;-F_{z 2} ;-F_{y 2}\right]^{T}
$$

with $F_{z 1}, F_{y 1}, F_{z 2}, F_{y 2}$ according to (21).

The excitation vector $\mathbf{f}$ can be split into sine and cosine components:

$\mathbf{f}=\sum_{k=1}^{2}\left\{\widehat{\mathbf{f}}_{k, \cos } \cdot \cos \left(2 \Omega \cdot t+2 \varphi_{k}\right)+\widehat{\mathbf{f}}_{k, \sin } \cdot \sin \left(2 \Omega \cdot t+2 \varphi_{k}\right)\right\}$,

with the amplitude vectors:

$$
\begin{aligned}
& \widehat{\mathbf{f}}_{1, \mathrm{cos}}=\left[0 ; 0 ; \widehat{F}_{z 1, \mathrm{cos}} ; \widehat{F}_{y 1, \mathrm{cos}} ; 0 ; 0 ;-\widehat{F}_{z 1, \cos } ;-\widehat{F}_{y 1, \cos } ; 0 ; 0\right]^{T} \text {, } \\
& \widehat{\mathbf{f}}_{1, \sin }=\left[0 ; 0 ; \widehat{F}_{z 1, \sin } ; \widehat{F}_{y 1, \sin } ; 0 ; 0 ;-\widehat{F}_{z 1, \sin } ;-\widehat{F}_{y 1, \sin } ; 0 ; 0\right]^{T} \text {, } \\
& \widehat{\mathbf{f}}_{2, \mathrm{cos}}=\left[0 ; 0 ; 0 ; 0 ; \widehat{F}_{z 2, \mathrm{cos}} ; \widehat{F}_{y 2, \mathrm{cos}} ; 0 ; 0 ;-\widehat{F}_{z 2, \cos } ;-\widehat{F}_{y 2, \cos }\right]^{T} \text {, }
\end{aligned}
$$

and the corresponding amplitudes

$$
\begin{gathered}
\hat{F}_{z k, \cos }=\Delta_{k} \cdot\left[-c_{z z k} \cdot \cos \beta_{k}-c_{z y k} \cdot \sin \beta_{k}\right], \\
\hat{F}_{y k, \cos }=\Delta_{k} \cdot\left[-c_{y z k} \cdot \cos \beta_{k}-c_{y y k} \cdot \sin \beta_{k}\right], \\
\hat{F}_{z k, \sin }=\Delta_{k} \cdot 2 \Omega \cdot\left[d_{z z k} \cdot \cos \beta_{k}+d_{z y k} \cdot \sin \beta_{k}\right], \\
\hat{F}_{y k, \sin }=\Delta_{k} \cdot 2 \Omega \cdot\left[d_{y z k} \cdot \cos \beta_{k}+d_{y y k} \cdot \sin \beta_{k}\right] .
\end{gathered}
$$

5.2. Solution. Referring to Gasch et al. [5], the following formulation is chosen to solve the differential equation:

$$
\begin{aligned}
& \mathbf{q}^{(n)}=\hat{\mathbf{q}}_{\cos }^{(n)} \cdot \cos \left(2 \Omega \cdot t+2 \varphi_{n}\right)+\hat{\mathbf{q}}_{\mathrm{sin}}^{(n)} \cdot \sin \left(2 \Omega \cdot t+2 \varphi_{n}\right) \quad \text { with: } n=1,2, \\
& \text { with: } \hat{\mathbf{q}}^{(n)}=\left[\hat{z}_{w}^{(n)} ; \hat{y}_{w}^{(n)} ; \hat{z}_{v 1}^{(n)} ; \hat{y}_{v 1}^{(n)} ; \hat{z}_{v 2}^{(n)} ; \hat{y}_{v 2}^{(n)} ; \hat{z}_{b 1}^{(n)} ; \hat{y}_{b 1}^{(n)} ; \hat{z}_{b 2}^{(n)} ; \hat{y}_{b 2}^{(n)}\right]^{T}, \\
& \hat{\mathbf{q}}_{\cos }^{(n)}=\left[\hat{z}_{w, \cos }^{(n)} ; \hat{y}_{w, \cos }^{(n)} ; \hat{z}_{v 1, \cos }^{(n)} ; \hat{y}_{v 1, \cos }^{(n)} ; \hat{z}_{v 2, \cos }^{(n)} ; \hat{y}_{v 2, \cos }^{(n)} ; \hat{z}_{b 1, \cos }^{(n)} ; \hat{y}_{b 1, \cos }^{(n)} ; \hat{z}_{b 2, \cos }^{(n)} ; \hat{y}_{b 2, \cos }^{(n)}\right]^{T}, \\
& \hat{\mathbf{q}}_{\sin }^{(n)}=\left[\hat{z}_{w, \sin }^{(n)} ; \hat{y}_{w, \sin }^{(n)} ; \hat{z}_{v 1, \sin }^{(n)} ; \hat{y}_{v 1, \sin }^{(n)} ; \hat{z}_{v 2, \sin }^{(n)} ; \hat{y}_{v 2, \sin }^{(n)} ; \hat{z}_{b 1, \sin }^{(n)} ; \hat{y}_{b 1, \sin }^{(n)} ; \hat{z}_{b 2, \sin }^{(n)} ; \hat{y}_{b 2, \sin }^{(n)}\right]^{T} .
\end{aligned}
$$


Here, index $n$ describes which shaft journal is causing the excitation. For example, if there is only excitation $s_{1}(t)$ from the shaft journal (1)-which means $s_{2}(t)=0 \rightarrow F_{z 2}=F_{y 2}=$ 0 - then only the forces $F_{z 1}$ and $F_{y 1}$ exist. In this case the index $n$ becomes $n=1$. If only an excitation $s_{2}(t)$ from the shaft journal (2) occurs, the index $n$ becomes $n=2$. In this context, index $k$ means the position that is being analyzed. The following example shows the correlation:

$$
z_{v k}^{(n)} \longrightarrow\left\{\begin{array}{l}
z_{v 1}^{(1)}: \text { vertical displacement of shaft journal }(1) \longrightarrow(k=1) \text {, caused by } s_{1}(t) \longrightarrow(n=1) \\
z_{v 1}^{(2)}: \text { vertical displacement of shaft journal }(1) \longrightarrow(k=1), \text { caused by } s_{2}(t) \longrightarrow(n=2) \\
z_{v 2}^{(1)}: \text { vertical displacement of shaft journal }(2) \longrightarrow(k=2), \text { caused by } s_{1}(t) \longrightarrow(n=1) \\
z_{v 2}^{(2)}: \text { vertical displacement of shaft journal }(2) \longrightarrow(k=2), \text { caused by } s_{2}(t) \longrightarrow(n=2) .
\end{array}\right.
$$

The single formulation (31) leads to the following matrix equation:

$$
\left[\begin{array}{cc}
\mathbf{C}-(2 \Omega)^{2} \cdot \mathbf{M} & 2 \Omega \cdot \mathbf{D} \\
-2 \Omega \cdot \mathbf{D} & \mathbf{C}-(2 \Omega)^{2} \cdot \mathbf{M}
\end{array}\right] \cdot\left[\begin{array}{c}
\hat{\mathbf{q}}_{\cos }^{(n)} \\
\hat{\mathbf{q}}_{\sin }^{(n)}
\end{array}\right]=\left[\begin{array}{c}
\hat{\mathbf{f}}_{k, \cos } \\
\hat{\mathbf{f}}_{k, \sin }
\end{array}\right]
$$

By solving this matrix equations for each single excitation, the amplitude vectors $\widehat{\mathbf{q}}_{\text {cos }}^{(n)}$ and $\widehat{\mathbf{q}}_{\text {sin }}^{(n)}$ can be computed and the solutions superposed:

$$
\begin{aligned}
& \mathbf{q}=\sum_{n=1}^{2} \mathbf{q}^{(n)}=\sum_{n=1}^{2}\left\{\hat{\mathbf{q}}_{\mathrm{cos}}^{(n)} \cdot \cos \left(2 \Omega \cdot t+2 \varphi_{n}\right)\right. \\
& \left.+\widehat{\mathbf{q}}_{\sin }^{(n)} \cdot \sin \left(2 \Omega \cdot t+2 \varphi_{n}\right)\right\} .
\end{aligned}
$$

5.3. Absolute Orbits. To describe the orbit movement of each point $\left(W, V_{1}, V_{2}, B_{1}, B_{2}\right)$ the complex coordinate systems in Figure 5 are now used, referring to Gasch et al. [5]. Index $\chi$ is used for the complex pointers $\underline{r}_{\chi}$, with

$$
\underline{r}_{\chi}=z_{\chi}+y_{\chi} \cdot j \quad \text { with: } \chi=w, v 1, v 2, b 1, b 2 .
$$

The solutions $\underline{r}_{\chi}$ can be described by two counterrotating vectors, one complex vector rotating in the direction of rotor rotation $(+2 \Omega)$ and one rotating opposite to the direction of rotor rotation $(-2 \Omega)$ :

$$
\underline{r}_{\chi}=e^{j \cdot 2 \Omega \cdot t} \cdot\left[\operatorname{Re}_{\chi}^{+}+j \cdot \operatorname{Im}_{\chi}^{+}\right]+e^{-j \cdot 2 \Omega \cdot t} \cdot\left[\operatorname{Re}_{\chi}^{-}+j \cdot \operatorname{Im}_{\chi}^{-}\right]
$$

with the real parts and the imaginary parts as follows:

$$
\begin{gathered}
\operatorname{Re}_{\chi}^{+}=\frac{1}{2} \cdot \sum_{n=1}^{2}\left[\cos \left(2 \varphi_{n}\right) \cdot\left(\hat{z}_{\chi, \cos }^{(n)}+\hat{y}_{\chi, \sin }^{(n)}\right)\right. \\
\left.-\sin \left(2 \varphi_{n}\right) \cdot\left(\hat{y}_{\chi, \cos }^{(n)}-\hat{z}_{\chi, \sin }^{(n)}\right)\right], \\
\operatorname{Im}_{\chi}^{+}=\frac{1}{2} \cdot \sum_{n=1}^{2}\left[\cos \left(2 \varphi_{n}\right) \cdot\left(\hat{y}_{\chi, \cos }^{(n)}-\hat{z}_{\chi, \sin }^{(n)}\right)\right. \\
\left.+\sin \left(2 \varphi_{n}\right) \cdot\left(\hat{z}_{\chi, \cos }^{(n)}+\hat{y}_{\chi, \sin }^{(n)}\right)\right], \\
\operatorname{Re}_{\chi}^{-}=\frac{1}{2} \cdot \sum_{n=1}^{2}\left[\cos \left(2 \varphi_{n}\right) \cdot\left(\hat{z}_{\chi, \cos }^{(n)}-\hat{y}_{\chi, \sin }^{(n)}\right)\right. \\
\left.\quad+\sin \left(2 \varphi_{\mathrm{n}}\right) \cdot\left(\hat{y}_{\chi, \cos }^{(n)}+\hat{z}_{\chi, \sin }^{(n)}\right)\right], \\
\operatorname{Im}_{\chi}^{-}=\frac{1}{2} \cdot \sum_{n=1}^{2}\left[\cos \left(2 \varphi_{n}\right) \cdot\left(\hat{y}_{\chi, \cos }^{(n)}+\hat{z}_{\chi, \sin }^{(n)}\right)\right. \\
\left.-\sin \left(2 \varphi_{n}\right) \cdot\left(\hat{z}_{\chi, \cos }^{(n)}-\hat{y}_{\chi, \sin }^{(n)}\right)\right] .
\end{gathered}
$$

The solution can also be described by the absolute values and by the phases

$$
\begin{gathered}
\underline{r}_{\chi}=\hat{r}_{\chi}^{+} \cdot e^{j \cdot \alpha_{\chi}^{+}} \cdot e^{j \cdot 2 \Omega \cdot t}+\hat{r}_{\chi}^{-} \cdot e^{j \cdot \alpha_{\chi}^{-}} \cdot e^{-j \cdot 2 \Omega \cdot t} \quad \text { with } \\
\hat{r}_{\chi}^{+}=\sqrt{\left(\operatorname{Re}_{\chi}^{+}\right)^{2}+\left(\operatorname{Im}_{\chi}^{+}\right)^{2}}, \quad \hat{r}_{\chi}^{-}=\sqrt{\left(\operatorname{Re}_{\chi}^{-}\right)^{2}+\left(\operatorname{Im}_{\chi}^{-}\right)^{2}},
\end{gathered}
$$

$$
\alpha_{\chi}^{+}:\left\{\begin{array}{l}
\operatorname{Re}_{\chi}^{+}>0 \longrightarrow \alpha_{\chi}^{+}=\arctan \left(\frac{\operatorname{Im}_{\chi}^{+}}{\operatorname{Re}_{\chi}^{+}}\right) \\
\operatorname{Re}_{\chi}^{+}<0 \longrightarrow \alpha_{\chi}^{+}=\pi+\arctan \left(\frac{\operatorname{Im}_{\chi}^{+}}{\operatorname{Re}_{\chi}^{+}}\right) \\
\operatorname{Re}_{\chi}^{+}=0 \wedge\left\{\begin{array}{l}
\operatorname{Im}_{\chi}^{+}>0 \longrightarrow \alpha_{\chi}^{+}=\frac{\pi}{2} \\
\operatorname{Im}_{\chi}^{+}<0 \longrightarrow \alpha_{\chi}^{+}=-\frac{\pi}{2},
\end{array}\right.
\end{array}\right.
$$




$$
\alpha_{\chi}^{-}:\left\{\begin{array}{l}
\operatorname{Re}_{\chi}^{-}>0 \longrightarrow \alpha_{\chi}^{-}=\arctan \left(\frac{\operatorname{Im}_{\chi}^{-}}{\operatorname{Re}_{\chi}^{-}}\right) \\
\operatorname{Re}_{\chi}^{-}<0 \longrightarrow \alpha_{\chi}^{-}=\pi+\arctan \left(\frac{\operatorname{Im}_{\chi}^{-}}{\operatorname{Re}_{\chi}^{-}}\right) \\
\operatorname{Re}_{\chi}^{-}=0 \wedge\left\{\begin{array}{l}
\operatorname{Im}_{\chi}^{-}>0 \longrightarrow \alpha_{\chi}^{-}=\frac{\pi}{2} \\
\operatorname{Im}_{\chi}^{-}<0 \longrightarrow \alpha_{\chi}^{-}=-\frac{\pi}{2} .
\end{array}\right.
\end{array}\right.
$$

The orbit shape of each point $\left(W, V_{1}, V_{2}, B_{1}, B_{2}\right)$ can be described by the ellipse parameters with the semimajor axis $a_{\chi}$, the semiminor axis $b_{\chi}$, and the angle of the major axis $\Psi_{\chi}$ (Figure 6):

$$
\begin{gathered}
a_{\chi}=\hat{r}_{\chi}^{+}+\hat{r}_{\chi}^{-}, \quad b_{\chi}=\left|\hat{r}_{\chi}^{+}-\hat{r}_{\chi}^{-}\right|, \\
\psi_{\chi}=\frac{\left(\alpha_{\chi}^{+}+\alpha_{\chi}^{-}\right)}{2} .
\end{gathered}
$$

5.4. Relative Orbits. To evaluate the vibration behavior, it is necessary to know not only the absolute orbits in a fixed coordinate system, but also the relative orbits between the bearing housing points $B_{k}$ and the shaft journal points $V_{k}$, to identify whether the oil film in the sleeve bearings could be critically disturbed. Therefore, vector $\underline{r}_{b k-v k}$ describes the relative orbit between the shaft journal points $V_{k}$ and the bearing housing points $B_{k}$ :

$$
\begin{aligned}
\underline{r}_{b k-v k}= & \underline{r}_{b k}-\underline{r}_{v k}=\hat{r}_{b k-v k}^{+} \cdot e^{j \cdot \alpha_{b k-v k}^{+}} \cdot e^{j \cdot 2 \Omega \cdot t} \\
& +\hat{r}_{b k-v k}^{-} \cdot e^{j \cdot \alpha_{b k-v k}^{-}} \cdot e^{-j \cdot 2 \Omega \cdot t}
\end{aligned}
$$

with

$$
\begin{aligned}
& \hat{r}_{b k-v k}^{+}= \sqrt{\left(\operatorname{Re}_{b k}^{+}-\operatorname{Re}_{v k}^{+}\right)^{2}+\left(\operatorname{Im}_{b k}^{+}-\operatorname{Im}_{v k}^{+}\right)^{2}}, \\
& \hat{r}_{b k-v k}^{-}=\sqrt{\left(\operatorname{Re}_{b k}^{-}-\operatorname{Re}_{v k}^{-}\right)^{2}+\left(\operatorname{Im}_{b k}^{-}-\operatorname{Im}_{v k}^{-}\right)^{2}}, \\
& \alpha_{b k-v k}^{+}:\left\{\begin{array}{l}
\left(\operatorname{Re}_{b k}^{+}-\operatorname{Re}_{v k}^{+}\right)>0 \longrightarrow \alpha_{b k-v k}^{+} \\
=\arctan \left(\frac{\operatorname{Im}_{b k}^{+}-\operatorname{Im}_{v k}^{+}}{\operatorname{Re}_{b k}^{+}-\operatorname{Re}_{v k}^{+}}\right) \\
\left(\operatorname{Re}_{b k}^{+}-\operatorname{Re}_{v k}^{+}\right)<0 \longrightarrow \alpha_{b k-v k}^{+} \\
\left(\operatorname{Re}_{b k}^{+}-\operatorname{Re}_{v k}^{+}\right) \\
=0 \wedge\left\{\begin{array}{l}
\left(\operatorname{Im}_{b k}^{+}-\operatorname{Im}_{v k}^{+}\right)>0 \longrightarrow \alpha_{b k-v k}^{+}=\frac{\pi}{2} \\
\left(\operatorname{Im}_{b k}^{+}-\operatorname{Im}_{v k}^{+}\right)<0 \longrightarrow \alpha_{b k-v k}^{+}=-\frac{\pi}{2}
\end{array}\right.
\end{array}\right.
\end{aligned}
$$

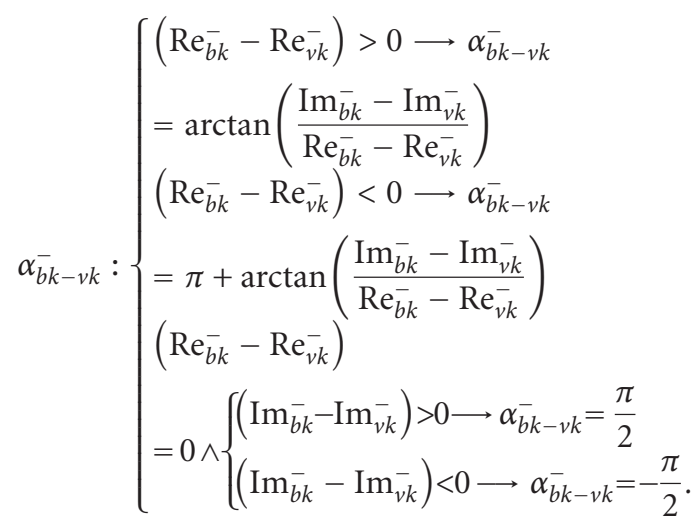

The relative orbit between the points $V_{1}$ and $B_{1}$ and between the points $V_{2}$ and $B_{2}$ can again be described by the ellipse parameters with the semi-major axis $a_{b k-v k}$, the semi-minor axis $b_{b k-v k}$, and the angle of the major axis $\Psi_{b k-v k}$ :

$$
\begin{gathered}
a_{b k-v k}=\hat{r}_{b k-v k}^{+}+\hat{r}_{b k-v k}^{-}, \quad b_{b k-v k}=\left|\hat{r}_{b k-v k}^{+}-\hat{r}_{b k-v k}^{-}\right|, \\
\psi_{b k-v k}=\frac{\left(\alpha_{b k-v k}^{+}+\alpha_{b k-v k}^{-}\right)}{2} .
\end{gathered}
$$

5.5. Bearing Housing Vibrations. In addition to the relative shaft displacements-described by the relative orbitsbetween the shaft journals and the bearing housings, also the vibration velocities of the bearing housings are often used in practice to evaluate the vibration quality of rotating machinery. To derive the vibration velocity of the bearing housing the horizontal and vertical displacement of the bearing housing points $B_{k}$ have to be known. By solving the differential equation system (23) the vertical displacement $z_{b k}$ and horizontal displacement $y_{b k}$ of the bearing house points $B_{k}$ can be described by

$$
\begin{aligned}
\delta_{b k}=\sum_{n=1}^{2}\{ & \hat{\delta}_{b k, \cos }^{(n)} \cdot \cos \left(2 \Omega \cdot t+2 \varphi_{n}\right) \\
& \left.+\hat{\delta}_{b k, \sin }^{(n)} \cdot \sin \left(2 \Omega \cdot t+2 \varphi_{n}\right)\right\} \quad \text { with } \delta=z, y .
\end{aligned}
$$

By differentiating (44) with respect to time, the vibration velocities of the bearing housing points $B_{k}$ can be derived:

$$
\begin{aligned}
& \dot{\delta}_{b k}=2 \Omega \cdot \sum_{n=1}^{2}\{-\hat{\delta}_{b k, \cos }^{(n)} \cdot \sin \left(2 \Omega \cdot t+2 \varphi_{n}\right) \\
&\left.+\hat{\delta}_{b k, \sin }^{(\mathrm{n})} \cdot \cos \left(2 \Omega \cdot t+2 \varphi_{n}\right)\right\} \\
&=2 \Omega \cdot \hat{\delta}_{b k} \cdot \sin \left(2 \Omega \cdot t+\alpha_{b k \delta}\right)
\end{aligned}
$$




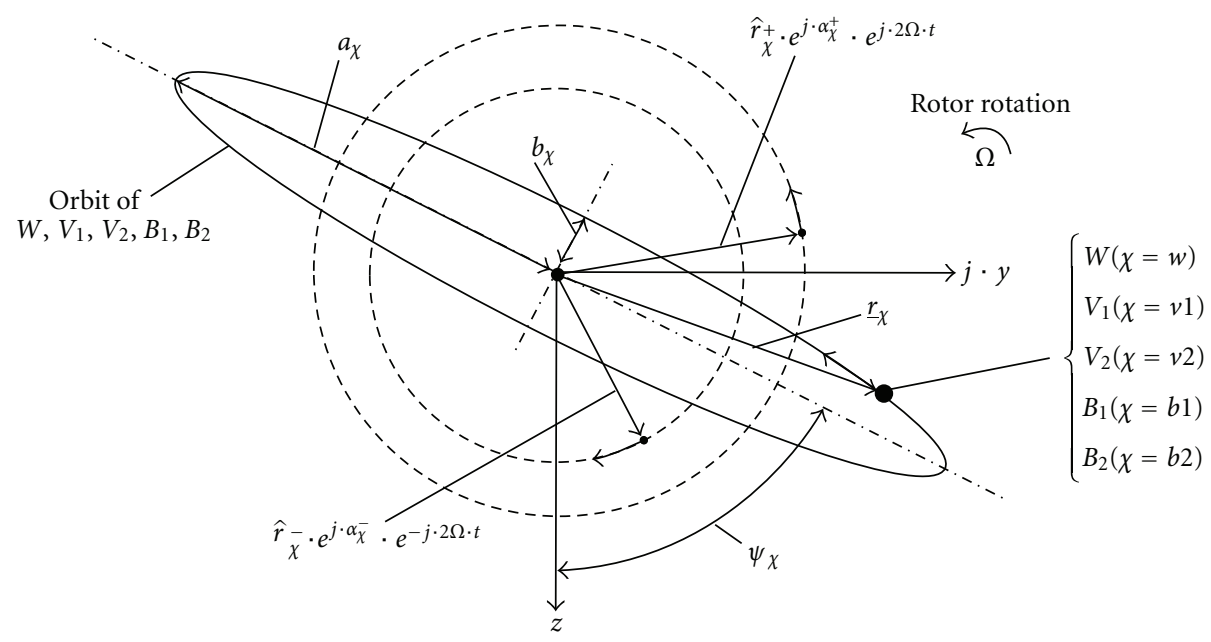

Notation: the orbits of $W, V_{1}, V_{2}, B_{1}, B_{2}$ are generally not the same; however, in this case, the same diagram is used for all of them.

FIgURE 6: Absolute orbits of the points $W, V_{1}, V_{2}, B_{1}, B_{2}$, described by ellipse parameters $a_{\chi}$, $b_{\chi}$, and $\Psi_{\chi}$.

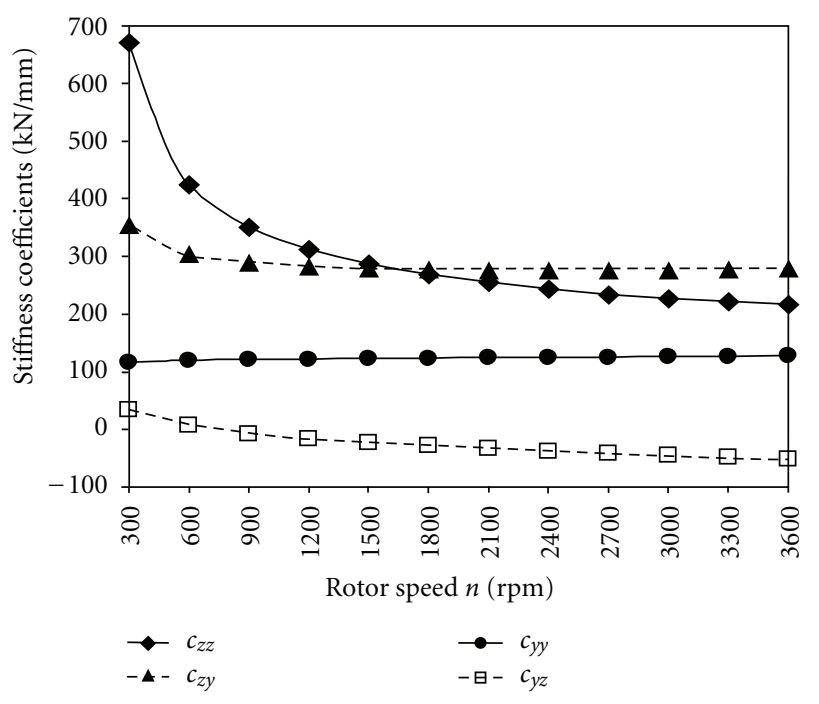

(a)

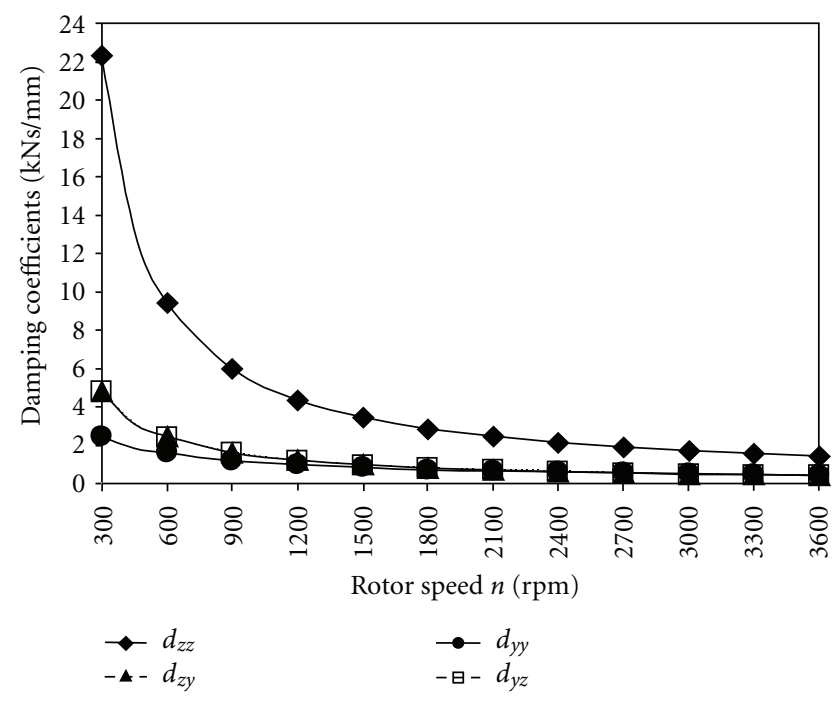

(b)

Figure 7: (a) Oil film stiffness coefficients. (b) Oil film damping coefficients.

with

$$
\begin{aligned}
& \hat{\delta}_{b k}^{(n)}=\sqrt{\left(-\hat{\delta}_{b k, \mathrm{cos}}^{(n)}\right)^{2}+\left(\hat{\delta}_{b k, \mathrm{sin}}^{(n)}\right)^{2}} \\
& \tan \alpha_{b k \delta}=\frac{\sum_{n=1}^{2} \hat{\delta}_{b k}^{(n)} \cdot \sin \left(2 \varphi_{n}+\alpha_{b k \delta}^{(n)}\right)}{\sum_{n=1}^{2} \hat{\delta}_{b k}^{(n)} \cdot \cos \left(2 \varphi_{n}+\alpha_{b k \delta}^{(n)}\right)}, \\
& \tan \alpha_{b k \delta}^{(n)}=\frac{\hat{\delta}_{b k, \mathrm{sin}}^{(n)}}{-\hat{\delta}_{b k, \mathrm{cos}}^{(n)}} .
\end{aligned}
$$

$\widehat{\delta}_{b k}$

$=\sqrt{\left(\sum_{n=1}^{2} \hat{\delta}_{b k}^{(n)} \cdot \cos \left(2 \varphi_{n}+\alpha_{b k \delta}^{(n)}\right)\right)^{2}+\left(\sum_{n=1}^{2} \hat{\delta}_{b k}^{(n)} \cdot \sin \left(2 \varphi_{n}+\alpha_{b k \delta}^{(n)}\right)\right)^{2}}$, 
Therefore the amplitudes of the vibration velocity of the bearing housing points $B_{k}$ can be described by

$$
\begin{gathered}
\text { Vertical: } \hat{v}_{b k z}=2 \Omega \cdot \hat{z}_{b k}, \\
\text { Horizontal: } \hat{v}_{b k y}=2 \Omega \cdot \hat{y}_{b k} .
\end{gathered}
$$

The amplitudes of the vibration velocities can also expressed by the real parts and the imaginary parts from Section 5.3 as follows:

$$
\text { Vertical: } \begin{aligned}
\hat{v}_{b k z} & =2 \Omega \cdot \hat{z}_{b k} \\
& =2 \Omega \cdot \sqrt{\left(\operatorname{Re}_{b k}^{+}+\operatorname{Re}_{b k}^{-}\right)^{2}+\left(-\operatorname{Im}_{b k}^{+}+\operatorname{Im}_{b k}^{-}\right)^{2}},
\end{aligned}
$$

Horizontal: $\hat{v}_{b k y}=2 \Omega \cdot \hat{y}_{b k}$

$$
=2 \Omega \cdot \sqrt{\left(\operatorname{Im}_{b k}^{+}+\operatorname{Im}_{b k}^{-}\right)^{2}+\left(\operatorname{Re}_{b k}^{+}-\mathrm{Re}_{b k}^{-}\right)^{2}} .
$$

\section{Example}

In this section the mathematical rotordynamic model is applied to a practical example. A two-pole induction motor with sleeve bearings (Figure 1)—with a power rating of $1 \mathrm{MW}$ and an operating speed range between $300 \mathrm{rpm}$ and $3600 \mathrm{rpm}$ - is analyzed. The absolute orbits of the shaft centre point $W$, the shaft journal points $V_{1}$ and $V_{2}$, and the bearing housing points $B_{1}$ and $B_{2}$ are calculated, as well as the relative orbits between the shaft journal points and the bearing housing points. The bearing housing vibration velocities are also calculated. The boundary conditions for the calculations are described in Section 6.1.

6.1. Boundary Conditions. The two-pole induction motor being investigated has a symmetrical design, which means here that the rotor core is placed symmetrically between the two sleeve bearings, so that the distances between the bearings and the centre of the rotor core are identical $\left(l_{1}=l_{2}\right)$ (Figure 4$)$. Also both sleeve bearings are identical. Therefore, the oil film stiffness and damping coefficients in both bearings are also identical (Figure 7) as well as the angle $\beta$ of the static displacement in the sleeve bearings (Figure 8 ).

Both diagrams (Figures 7 and 8) show that the oil film stiffness and damping coefficients and the angle of the static displacement $\beta$ are a function of the rotor speed $n$.

Both shaft journals (1) and (2) are assumed to be elliptical with a form deviation of $0.002 \%$ relative to the diameter $d$ of the shaft journal $(d=100 \mathrm{~mm})$ :

$$
\Delta_{1}=\Delta_{2}=2 \cdot 10^{-5} \cdot d=2 \mu \mathrm{m} .
$$

In the first consideration, the orientation of the elliptical shaft journals is assumed to be equal:

$$
\varphi_{1}=\varphi_{2}
$$

Subsequently, also different orientations of the elliptical shaft journals are considered. The angle $\Delta \varphi$ is the difference angle, which describes the different orientation of the elliptical shaft journals.

$$
\varphi_{2}=\varphi_{1}+\Delta \varphi \quad \text { with } \Delta \varphi=0 \cdots 90^{\circ} .
$$

With these boundary conditions, the absolute orbits of the shaft centre point $W$, the shaft journal points $V_{1}$ and $V_{2}$ and the bearing housing points $B_{1}$ and $B_{2}$ are calculated as well as the relative orbits between the shaft journal points $V_{1}$ and $B_{1}$ and $V_{2}$, and $B_{2}$. Additionally, also the vibration velocities at the bearing housing are calculated.

6.2. Absolute Orbits. In this section the absolute orbits of the shaft centre point $W$, the shaft journal points $V_{1}$ and $V_{2}$, and the bearing housing points $B_{1}$ and $B_{2}$ are calculated.

6.2.1. Absolute Orbits for Identical Orientation of the Elliptical Shaft Journals $\left(\varphi_{1}=\varphi_{2}\right)$. First, the absolute orbits of the shaft centre point $W$, the shaft journal points $V_{1}$ and $V_{2}$, and the bearing housing points $B_{1}$ and $B_{2}$ are calculated for identical orientation of the elliptical shaft journals $\left(\varphi_{1}=\varphi_{2}\right)$. In this case the absolute orbits of $V_{1}$ and $V_{2}$ are identical as well as the orbits of $B_{1}$ and $B_{2}$. The calculated semi-major axes, the semi-minor axes, and the angle of the semi-major axes are calculated and shown in Figure 9, together with the definition of the ellipse parameters of the absolute orbits.

Figure 9(a) shows that the maximum value of the semimajor axis $a_{w}(17.6 \mu \mathrm{m})$ of the shaft centre point $W$ is reached at a rotor speed of $2220 \mathrm{rpm}$. In contrast, the maximum values of the semi-major axes $a_{v 1}$ and $a_{v 2}$ of the shaft journal points $V_{1}$ and $V_{2}(4.7 \mu \mathrm{m})$ are reached at a rotor speed of $2180 \mathrm{rpm}$. The maximum values of the semi-major axes $a_{b 1}$ and $a_{b 2}$ of the bearing housing points $B_{1}$ and $B_{2}$ $(3.85 \mu \mathrm{m})$ are reached at a rotor speed of $2240 \mathrm{rpm}$. At a rotor speed of $2590 \mathrm{rpm}$ the semi-major axes of the shaft journal points $V_{1}$ and $V_{2}$ become zero. At this speed, also the semiminor axes for these orbits must get zero (Figure 9(b)). At the other speeds, where the semi-minor axes go to zero, the absolute orbits become straight lines. Figure 9(c) shows the angles of the semi-major axes. The angles of the semi-majors of the orbits of $V_{1}$ and $V_{2}$ and $W$ are identical $\left(\Psi_{v 1}=\Psi_{v 2}=\right.$ $\left.\Psi_{w}\right)$ and lie in a range between $18^{\circ}$ and $33^{\circ}$. The angles $\Psi_{b 1}$ and $\Psi_{b 2}$ of the semi-major axes of $B_{1}$ and $B_{2}$ lie in a range between $31^{\circ}$ and $51^{\circ}$.

\subsubsection{Absolute Orbits for Different Orientation of the Elliptical} Shaft Journals $\left(\varphi_{2}=\varphi_{1}+\Delta \varphi\right)$. In this section, only the behavior of the semi-major axis is analyzed, because this value is the most important value regarding the evaluation of the vibration behavior. Figure 10 shows the semi-major axis of the orbit of the shaft centre point $W$, depending on the rotor speed $n$ and the difference angle $\Delta \varphi$. The maximum value $(17.6 \mu \mathrm{m})$ is reached at a rotor speed of $2220 \mathrm{rpm}$ and a difference angle of $\Delta \varphi=0^{\circ}$.

For different orientation of the elliptical shaft journals $\left(\varphi_{2}=\varphi_{1}+\Delta \varphi\right)$ the orbits of $V_{1}$ and $V_{2}$ are no longer identical (Figures 11(a) and 11(b)), although a symmetrical design was chosen (see Section 6.1). The maximum value $(5.0 \mu \mathrm{m})$ for $a_{v 1}$ is reached at a rotor speed of $2180 \mathrm{rpm}$ and at a 


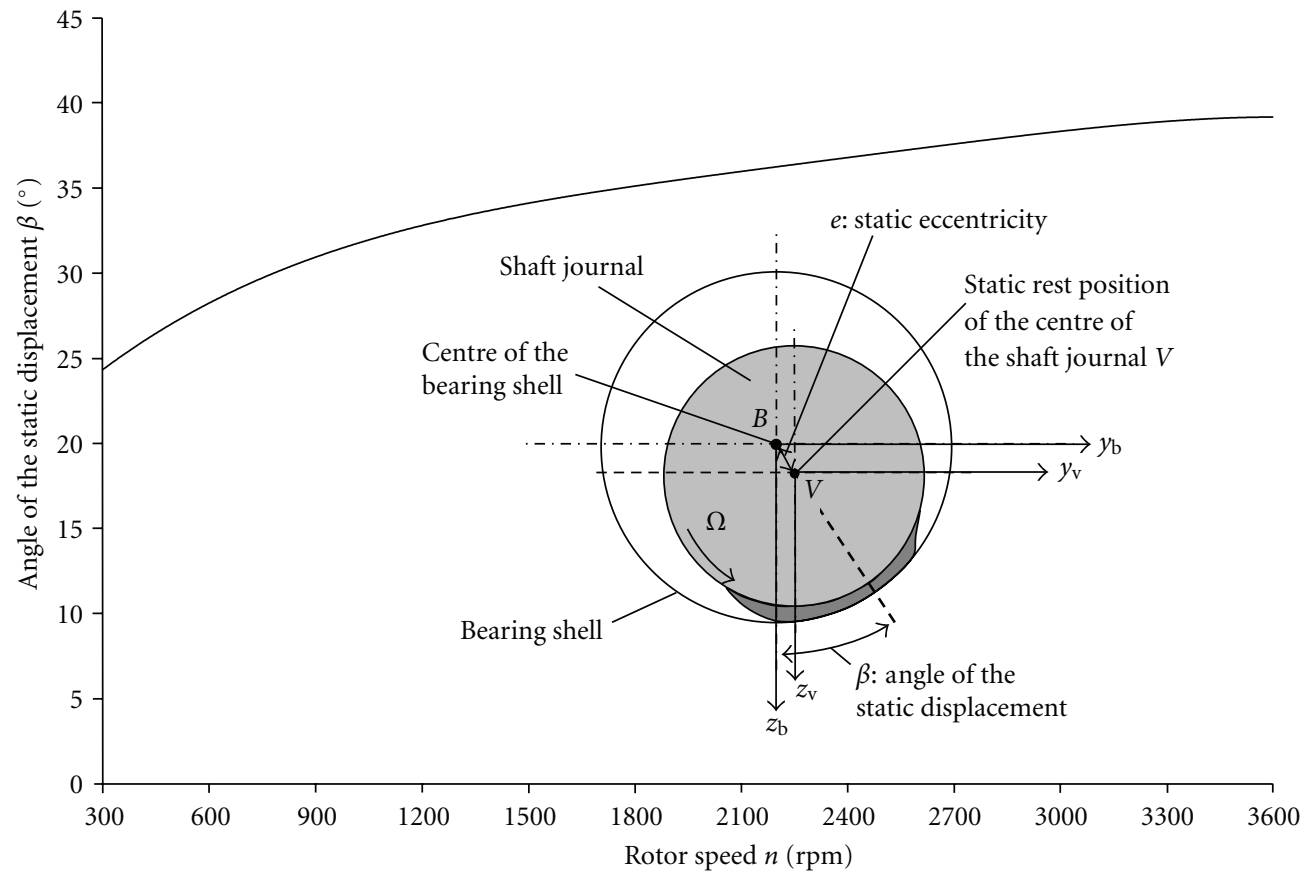

Figure 8: Angle $\beta$ of the static displacement in the sleeve bearings.

difference angle of $\Delta \varphi=22^{\circ}$. The maximum value $(4.7 \mu \mathrm{m})$ for $a_{v 2}$ is reached at the same rotor speed but at a difference angle of $\Delta \varphi=0^{\circ}$.

For a negative orientation $\varphi_{2}=\varphi_{1}-\Delta \varphi$ the characteristics of $a_{v 1}$ and $a_{v 2}$ will interchange. In contrast, the characteristics of $a_{b 1}$ and $a_{b 2}$ (Figures $11(\mathrm{c})$ and 11(d)) are identical. The maximum value $(3.85 \mu \mathrm{m})$ is reached at a rotor speed of $2240 \mathrm{rpm}$ and at a difference angle of $\Delta \varphi=0^{\circ}$.

6.3. Relative Orbits. After the absolute orbits are analyzed, the relative orbits between the bearing housing point $B_{1}$ and the shaft journal point $V_{1}$ and between the bearing housing point $B_{2}$ and the shaft journal point $V_{2}$ are analyzed in this section.

6.3.1. Relative Orbits for Identical Orientation of the Elliptical Shaft Journals $\left(\varphi_{1}=\varphi_{2}\right)$. First, the relative orbits between the bearing housing point $B_{1}$ and the shaft journal point $V_{1}$ and between the bearing housing point $B_{2}$ and the shaft journal point $V_{2}$ are analyzed for identical orientation of the elliptical shaft journals $\left(\varphi_{1}=\varphi_{2}\right)$. In this case the relative orbits between $V_{1}$ and $B_{1}$ and between $V_{2}$ and $B_{2}$ are again identical.

Figure 12(a) shows that the maximum value of the semimajor axes $a_{b 1-v 1}(2.07 \mu \mathrm{m})$ of the relative orbit between the bearing housing point $B_{1}$ and the shaft journal point $V_{1}$ is reached at a rotor speed of $1720 \mathrm{rpm}$. Figure 12(b) shows that at three rotor speeds-2130 rpm, $2260 \mathrm{rpm}$, and $2300 \mathrm{rpm}-$ the semi-minor axis $b_{b 1-v 1}$ becomes zero. At these speeds the relative orbit between the bearing housing point $B_{1}$ and the shaft journal point $V_{1}$ becomes a straight line.

Figure 12(c) shows that the angle $\Psi_{b 1-v 1}$ of the semimajor axis of the relative orbit lies in a range between $36^{\circ}$ and $211^{\circ}$.

6.3.2. Relative Orbits for Different Orientation of the Elliptical Shaft Journals $\left(\varphi_{2}=\varphi_{1}+\Delta \varphi\right)$. Figure 13(a) shows the semimajor axis $a_{b 1-v 1}$ of the relative orbit between the bearing housing point $B_{1}$ and $V_{1}$. The maximum value of $a_{b 1-v 1}$ $(2.4 \mu \mathrm{m})$ is reached at a rotor speed of $2100 \mathrm{rpm}$ and at a difference angle of $\Delta \varphi=48^{\circ}$.

The maximum value of $a_{b 2-v 2} \quad(2.2 \mu \mathrm{m})$-which describes the semi-major axis $a_{b 2-v 2}$ of the relative orbit between the bearing housing point $B_{2}$ und $V_{2}$ (Figure 13(b)) - is reached at a rotor speed of $2445 \mathrm{rpm}$ and at a difference angle of $\Delta \varphi=62^{\circ}$. For a negative orientation $\varphi_{2}=\varphi_{1}-\Delta \varphi$ the characteristics of $a_{b 1-v 1}$ and $a_{b 2-v 2}$ will interchange again. Therefore it is shown again that the vibration characteristic for bearing (1) and bearing (2) is different, if a difference angle $\Delta \varphi$ exists, although a symmetrical design was chosen.

6.4. Bearing Housing Vibrations. After the absolute orbits and the relative orbits have been analyzed, the bearing housing vibrations are now investigated in this section.

6.4.1. Bearing Housing Vibrations for Identical Orientation of the Elliptical Shaft Journals $\left(\varphi_{1}=\varphi_{2}\right)$. First, the bearing 


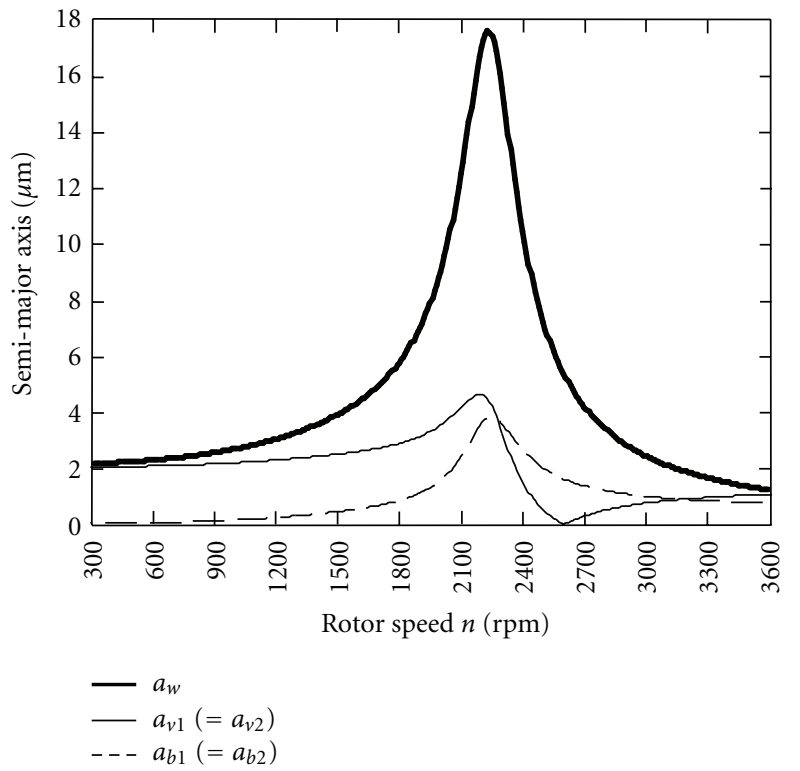

(a)

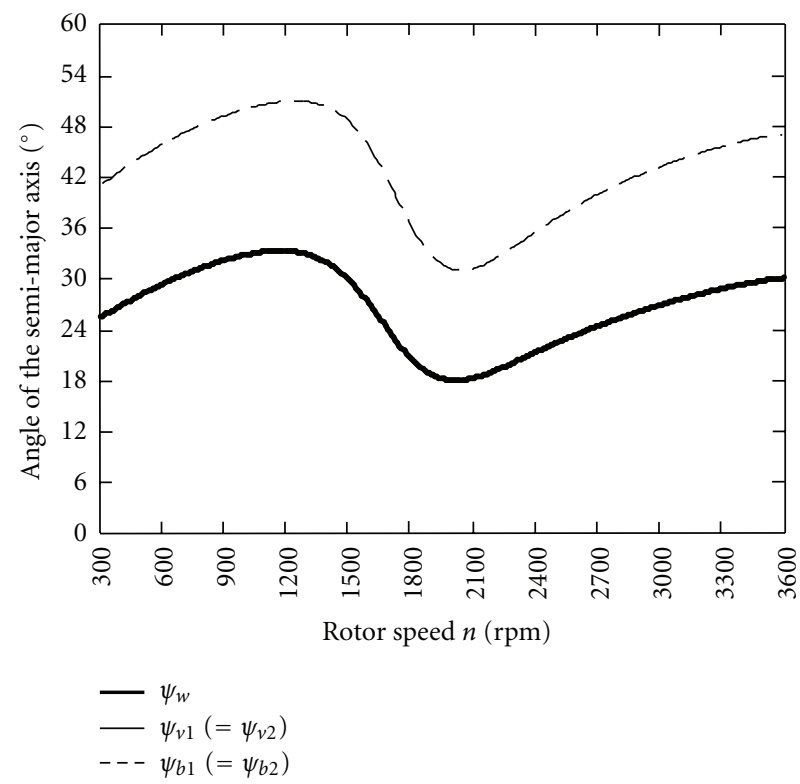

(c)

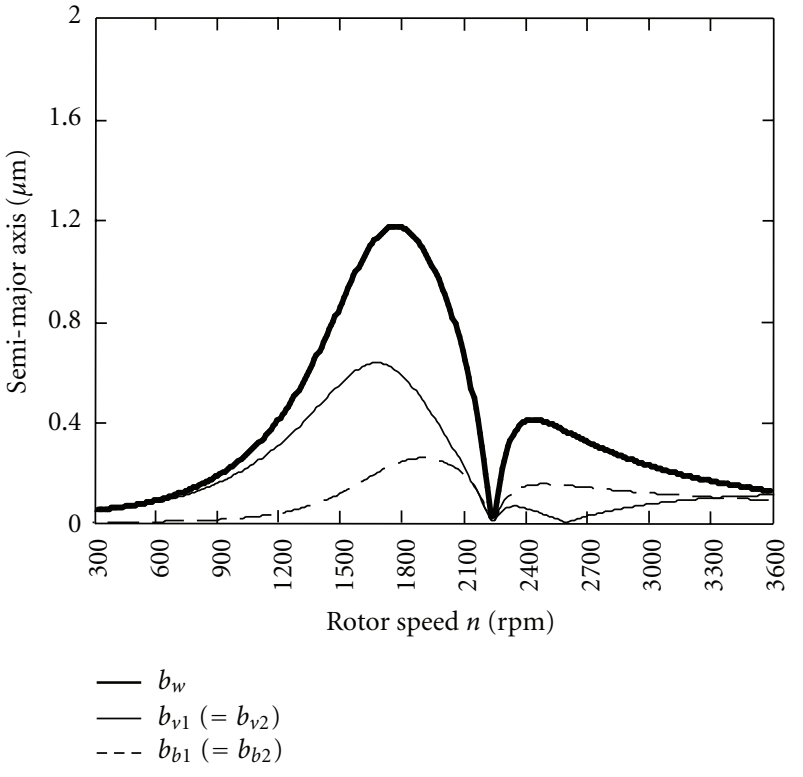

(b)

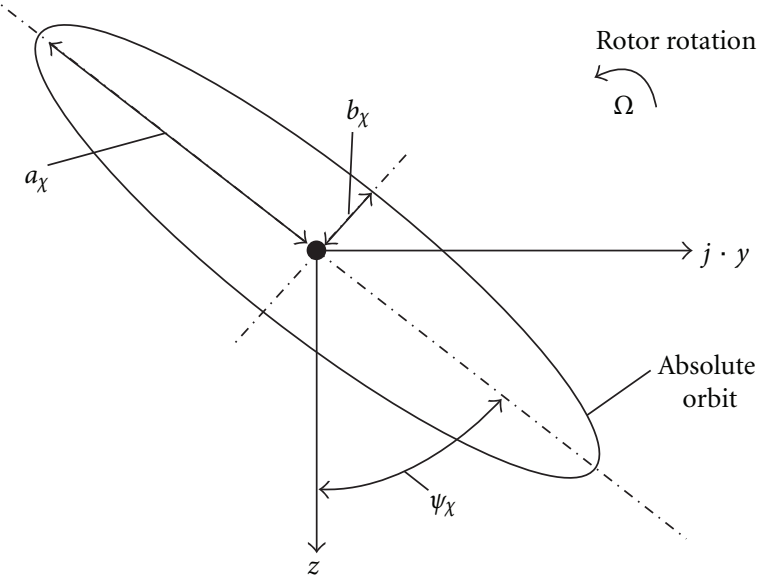

(d)

Figure 9: Absolute orbits of the shaft centre point $W$, of the shaft journal points $V_{1}$ and $V_{2}$ and of the bearing housing points $B_{1}$ and $B_{2}$, described by (a) the semi-major axes, (b) the semi-minor axes, and (c) the angles of the semi-major axes. (d) Definition of the absolute orbits. Boundary condition: identical orientation of the elliptical shaft journals $\left(\varphi_{1}=\varphi_{2}\right)$ and different rotor speeds.

housing vibrations are analyzed for identical orientation of the elliptical shaft journals $\left(\varphi_{1}=\varphi_{2}\right)$. In this case the bearing housing vibrations for both bearing housings are identical. Figure 14(a) shows that the maximum vibration velocity in the vertical direction $(1.52 \mathrm{~mm} / \mathrm{s})$ occurs at a rotor speed of $2240 \mathrm{rpm}$. The maximum vibration velocity in the horizontal direction $(0.98 \mathrm{~mm} / \mathrm{s})$ occurs at the same rotor speed.
6.4.2. Bearing Housing Vibrations for Different Orientation of the Elliptical Shaft Journals $\left(\varphi_{2}=\varphi_{1}+\Delta \varphi\right)$. Figures 15(a) and 15 (b) show the characteristics of the vibration velocity in the vertical direction at bearing housing (1) (Figure 15(a)) and at bearing housing (2) (Figure 15(b)). Both diagrams are identical. The maximum vibration velocity in the vertical direction $(1.52 \mathrm{~mm} / \mathrm{s})$ for the bearing housings is reached 


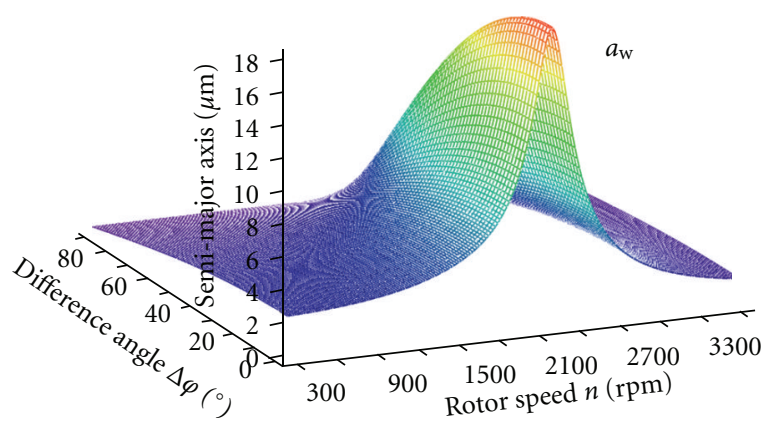

Figure 10: Semi-major axis $a_{w}$ of the absolute orbit of the shaft centre point $W$, depending on the rotor speed $n$ and on the difference angle $\Delta \varphi$.

at a rotor speed of $2240 \mathrm{rpm}$ and at a difference angle of $\Delta \varphi=0^{\circ}$.

Figures 15(c) and 15(d) show the characteristics of the vibration velocity in the horizontal direction at bearing housing (1) (Figure 15(c)) and at bearing housing (2) (Figure 15(d)). Again, both diagrams are identical. The maximum vibration velocity in the horizontal direction $(0.98 \mathrm{~mm} / \mathrm{s})$ for the bearing housings is also reached at a rotor speed of $2240 \mathrm{rpm}$ and at a difference angle of $\Delta \varphi=0^{\circ}$.

6.5. Evaluation of the Vibration Behavior. The rotordynamic model was applied to a two-pole induction motor, which has elliptical shaft journals with an arbitrarily chosen form deviation $\Delta$ of $0.002 \%$ relative to the diameter $d$ of the shaft journals. To evaluate the vibrations, the relative shaft vibrations between the shaft journals and the bearing housing have to be considered, as well as the bearing housing velocities.

The relative shaft displacements over the complete speed range $(300 \mathrm{rpm}$ to $3600 \mathrm{rpm}$ ) are very low-the maximum value of the semi-major axis of the relative orbit is only $2.4 \mu \mathrm{m}$ - so that only $3.1 \%$ of the radial bearing clearance of the sleeve bearings is captured by the relative shaft displacements. A critical disturbance of the oil film would be expected, if the relative shaft displacements would exceed more than $60 \%$ of the radial bearing clearance. Additionally the relative shaft displacements are also within the limit of the standard EN 60034-14 [16] -relative shaft displacements $<65 \mu \mathrm{m}$ (level A) peak-to-peak value and $<50 \mu \mathrm{m}$ (level B) peak-to-peak value-and even within the limits of API 541 [17] — shaft displacements $<38.1 \mu \mathrm{m}$ peak to peak value.

Also the bearing housing vibrations over the complete speed range fulfill the vibration limits, which are defined in the standard EN 60034-14 [16] and API 541 [17]. Here the effective bearing housing vibration for this kind should not exceed $2.3 \mathrm{~mm} / \mathrm{s} \mathrm{rms}$ (level A) and $1.5 \mathrm{~mm} / \mathrm{s} \mathrm{rms} \mathrm{(level}$ B) for EN 60034-14 and $2.54 \mathrm{~mm} / \mathrm{s}$ 0-peak for API 541. At the critical speed the maximum bearing housing vibrations is $1.52 \mathrm{~mm} / \mathrm{s}$ 0-peak, which corresponds to $1.07 \mathrm{~mm} / \mathrm{s} \mathrm{rms}$.
It is also possible to evaluate the movement of the shaft centre point $W$. The semi-major axis of the shaft centre point $W$ reaches the maximum value $(17.6 \mu \mathrm{m})$ at a rotor speed of $2220 \mathrm{rpm}$, which means $0.4 \%$ of the air gap of the motor. As a consequence, rubbing between rotor and stator can be completely ruled out.

In the paper the oil film stiffness and damping coefficients are calculated for the ideal (circular) shape of the shaft journal with the constant radius $r$ (Figure 3 ). This leads to a mean radial bearing clearance of $\mathrm{CR}=76.5 \mu \mathrm{m}$. But for the elliptical shaft journal a maximum radius of $r_{\max }=r+\Delta$ and minimum radius of $r_{\min }=r-\Delta$ occur $(\Delta=2 \mu \mathrm{m})$. When considering $r_{\max }$ and $r_{\min }$, the mean radial bearing clearance will only change marginal $( \pm 2.61 \%)$. Therefore also the oil film stiffness and damping coefficients will only change marginal. When calculating once with the bigger bearing clearance $\mathrm{CR}=78.5 \mu \mathrm{m}$ and once with the smaller bearing clearance $\mathrm{CR}=74.5 \mu \mathrm{m}$, the change of the maximum value of the semi-major axis $\left(a_{w}=17.6 \mu \mathrm{m}\right)$ of the shaft centre point $W$ (Figure $9(\mathrm{a})$ ) will only be about $\pm 2-3 \%$. Therefore the calculation of the oil film stiffness and damping coefficients with the ideal shape of the shaft journal is here sufficient enough.

The electromagnetic influence in the rotordynamic model is considered by using a magnetic spring $c_{m}$, which is a common simplification for electrical machines. In this paper, no electromagnetic field damping effects, for example, by the rotor cage, are considered and therefore only a radial magnetic pull, but no tangential magnetic force. A tangential magnetic force will become more important, when analyzing instabilities $[10,11,14]$. However for a forced vibration analysis, the worst case regarding the radial magnetic pull is considered in the example, because no field damping is considered as well as no saturation of the iron parts and no influence of the slots, which both would increase the effective electromagnetic air gap. Additionally, a maximum homopolar flux is also considered. All these boundary conditions lead to a worst case regarding the magnitude of the radial magnetic spring constant $c_{m}$. Although considering this worst case, the influence of the radial magnetic spring constant on the vibration is only marginal. The maximum value of the semi-major axis of the shaft centre point $W$, calculated with the worst case regarding the magnetic spring constant, is here $a_{w}=17.6 \mu \mathrm{m}$ (Figure 9(a), which is only $0.39 \%$ of the air gap width of the motor. Without electromagnetic influence $\left(c_{m}=0\right)$ the maximum value of the semi-major axis of the shaft centre point $W$ becomes $a_{w}=17.545 \mu \mathrm{m}$, which is only $0.31 \%$ lower. This shows that the electromagnetic influence is here very low.

In the paper, mass effects, for example, gyroscopic effects, are neglected. In most large induction motors the rotor core is more or less symmetrically positioned between the bearings - $l_{1} \approx l_{2}$ (Figures 1 (a) and 4 ) - and nearly the same rotor stiffness exists along $l_{1}$ and $l_{2}$, and if no large overhang mass at the shaft end is positioned, then the influence of the gyroscopic effect on the first critical bending frequency is usually very low. A finite element analysis regarding the natural frequencies of the here investigated rotor was performed, showing a first critical bending frequency of 


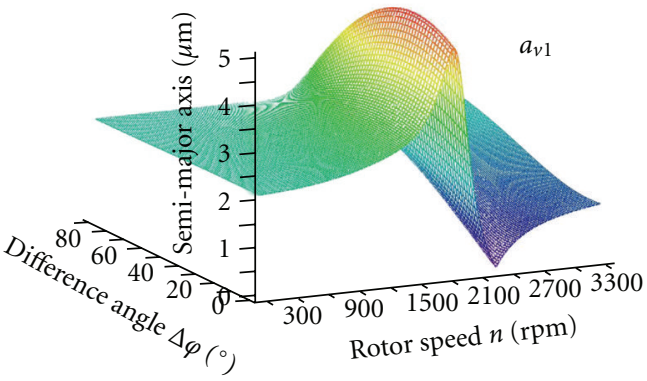

(a)

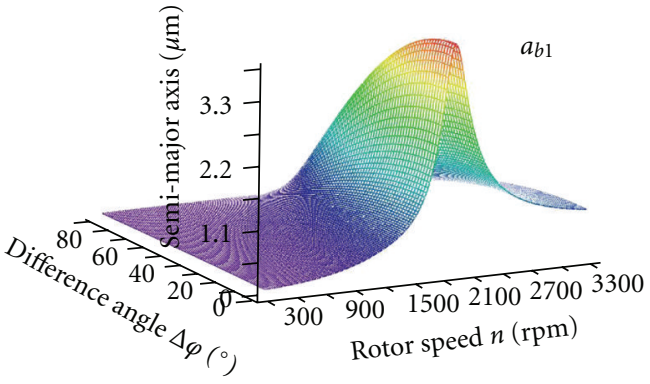

(c)

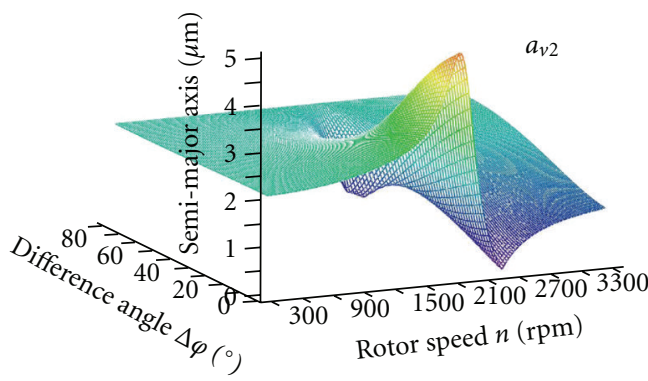

(b)

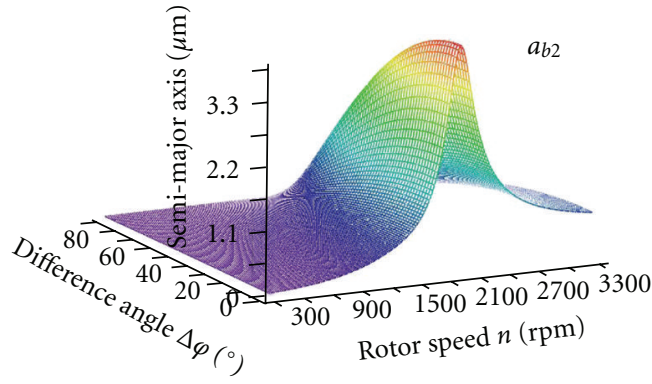

(d)

Figure 11: Semi-major axes of the absolute orbits of (a) the shaft journal point $V_{1}$, (b) the shaft journal point $V_{2}$, (c) the bearing housing point $B_{1}$, and (d) the bearing housing point $B_{2}$, depending on the rotor speed $n$ and on the difference angle $\Delta \varphi$.

$86.37 \mathrm{~Hz}$, when the rotor is supported in rigid bearings and does not rotate $(n=0 \mathrm{U} / \mathrm{min})$. When the rotor rotates at $3600 \mathrm{U} / \mathrm{min}$ the first critical bending frequency is split due to the gyroscopic effect into two critical bending frequencies $(86.11 \mathrm{~Hz}$ and $86.62 \mathrm{~Hz})$, supported in rigid bearings. This shows that the influence of the gyroscopic effect is only marginal and can be neglected here.

The here analyzed elliptical form deviation of $\Delta=$ $2 \mu \mathrm{m}$ would lead to a mechanical runout value of $4 \mu \mathrm{m}$, if no additional form deviation of the shaft journal occurs. Referring to the standard EN 60034-14 [16], the allowed combined runout (mechanical and electrical runout) should not exceed $16 \mu \mathrm{m}$ for Grande A. If for this limit no electrical run out would occur and the mechanical runout would be only caused by the elliptical form deviation-in this case the form deviation has to be $\Delta=8 \mu \mathrm{m}$-then the vibration values would be four times higher as calculated in this example, if linearization of the system may be assumed for such a large form deviation. But this form deviation of $\Delta=8 \mu \mathrm{m}$ is about $4-5$ times higher, as is usually seen in practice.

Notation. All of the calculations were only performed for excitation due to elliptical shaft journals, as this is the focus of the paper. Of course also other excitations, for example, mechanical unbalance or rotating unbalanced magnetic pull - which both occur with rotor angular frequency $(1 x)$ have to be considered in practice.

\section{Conclusion}

The paper shows a theoretical vibration analysis regarding excitation due to elliptical shaft journals in sleeve bearings of electrical motors, based on a simplified rotordynamic model. It is shown that elliptical shaft journals lead to kinematic constraints regarding the movement of the shaft journals on the oil film of the sleeve bearings and therefore to an excitation of the rotordynamic system. The rotordynamic system-which consists of the rotor, the oil film of the sleeve bearings and the structural stiffness of the sleeve bearings and of the end shields, the electromagnetic coupling between rotor and stator, and the excitation due to elliptical shaft journals - is described by a linear differential equation system. The solution of this differential equation system leads to the mathematical description of the movement of the rotor mass, the shaft journals, and the sleeve bearing housings. Additionally, the relative movements between the shaft journals and the bearing housings are deduced, as well as the bearing housing vibration velocities. To clarify the mathematical model an example of a two-pole induction 


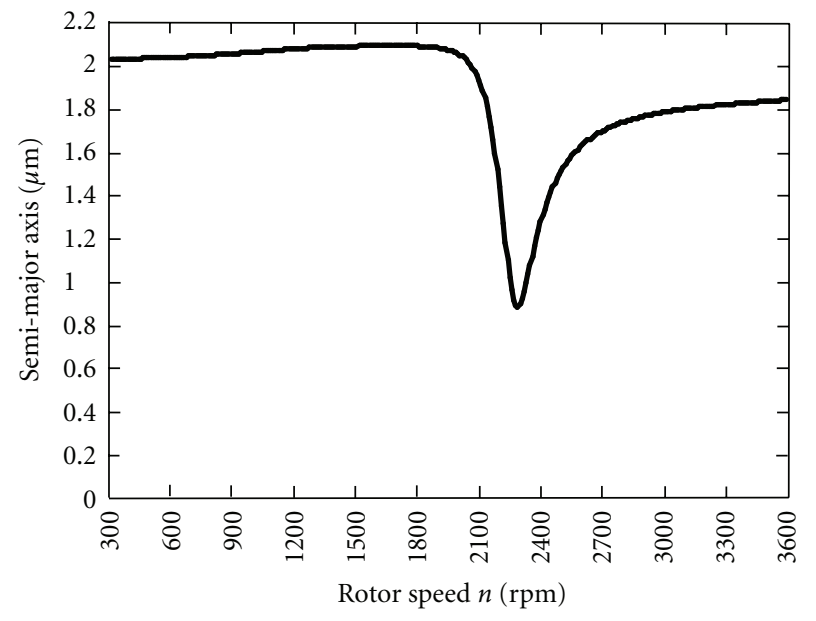

$-a_{b 1-v 1}\left(=a_{b 2-v 2}\right)$

(a)

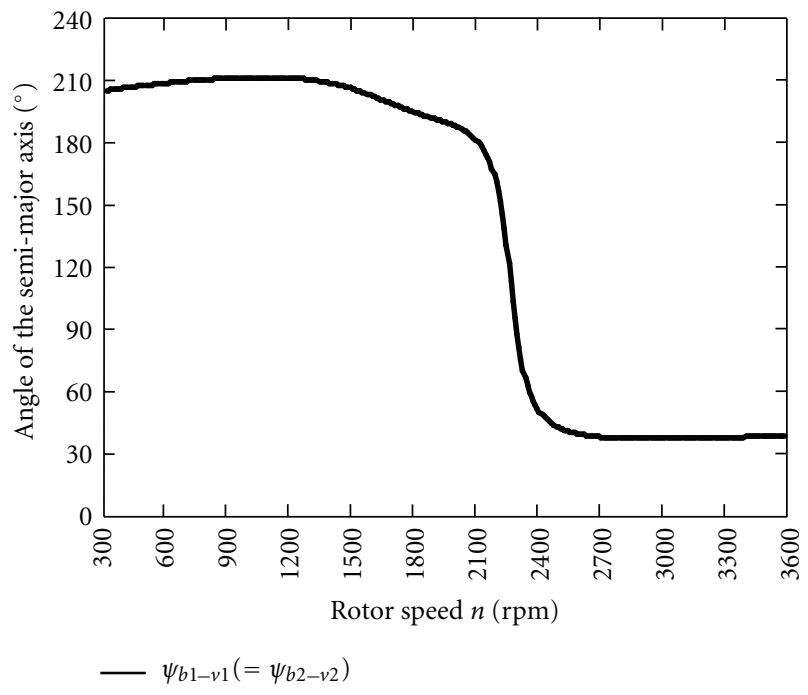

(c)

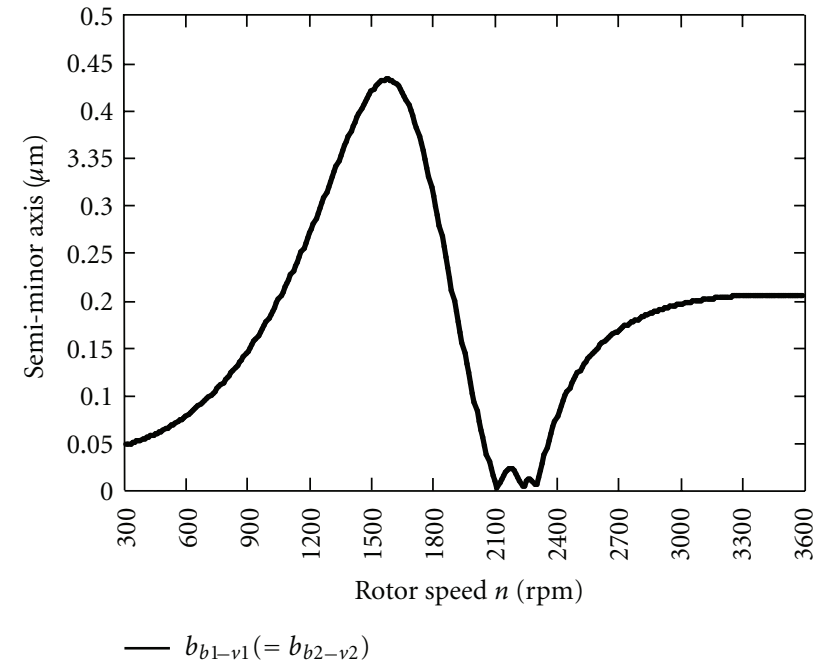

(b)

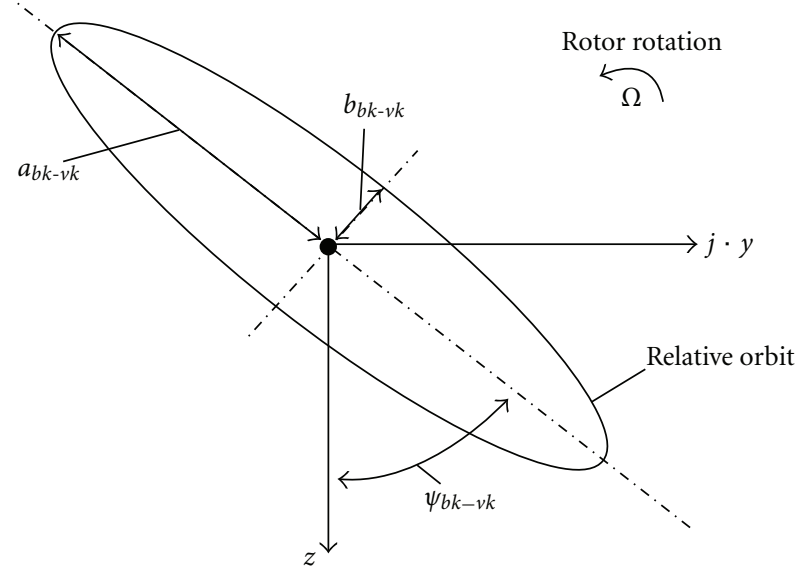

(d)

FIGURE 12: Relative orbits between the bearing housing point $B_{1}$ and the shaft journal point $V_{1}$ and between $B_{2}$ and $V_{2}$, described by (a) the semi-major axes, (b) the semi-minor axes, and (c) the angle of the semi-major axes. (d) Definition of the relative orbits. Boundary condition: identical orientation of the elliptical shaft journals $\left(\varphi_{1}=\varphi_{2}\right)$ and different rotor speeds $n$.

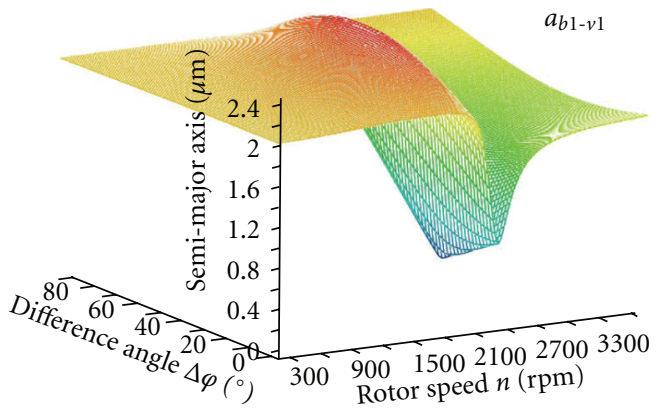

(a)

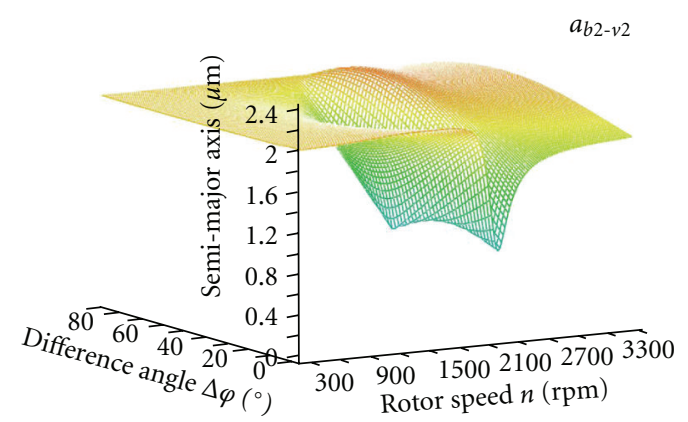

(b)

Figure 13: Semi-major axes of the relative orbit (a) between $B_{1}$ and $V_{1}$ and (b) between $B_{2}$ and $V_{2}$, depending on the rotor speed $n$ and on the difference angle $\Delta \varphi$. 


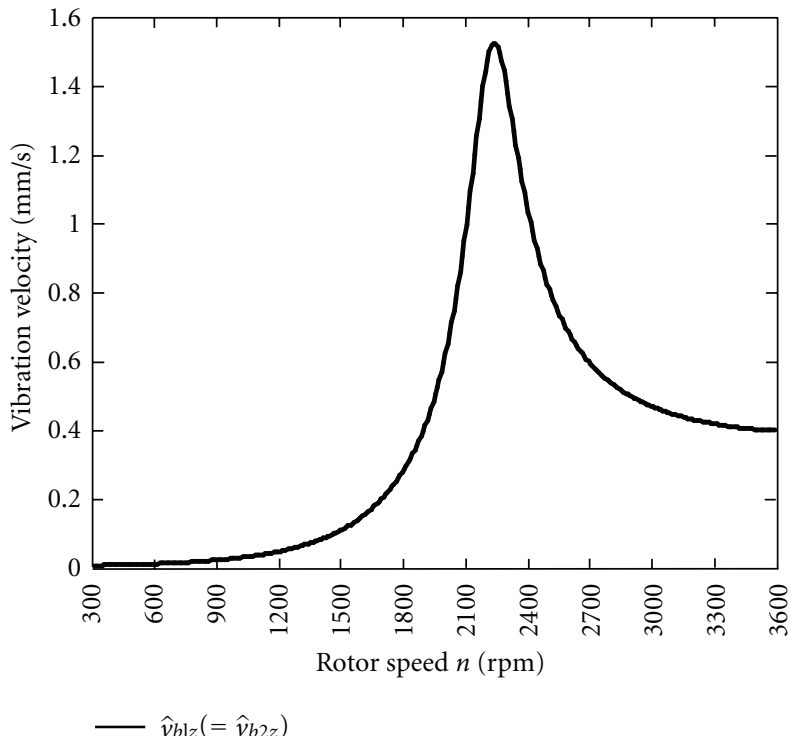

(a)

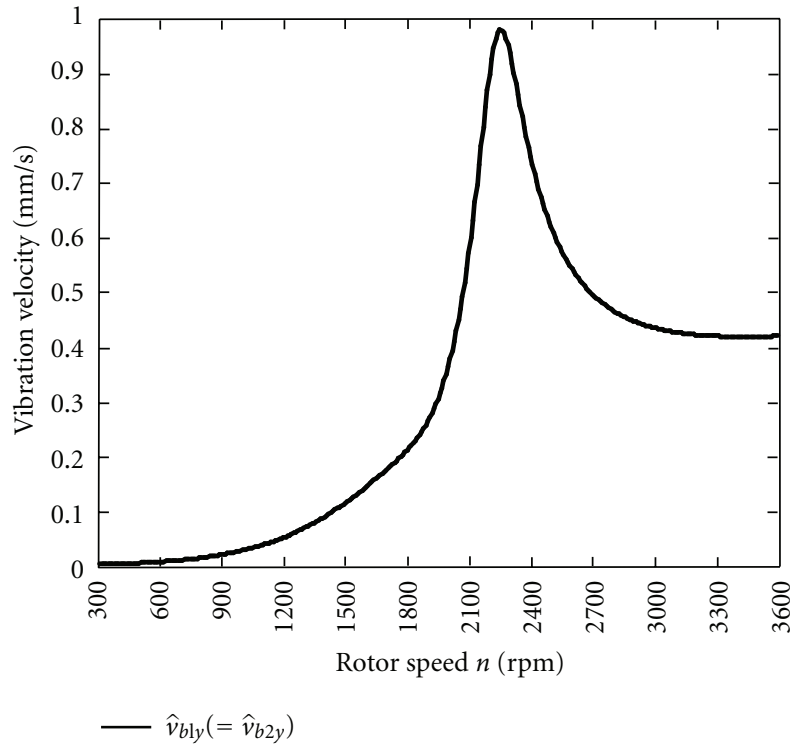

(b)

FIGURE 14: Bearing housing velocities in (a) vertical direction ( $z$-direction), (b) in horizontal direction ( $y$-direction). Boundary condition: identical orientation of the elliptical shaft journals $\left(\varphi_{1}=\varphi_{2}\right)$ and different rotor speeds $n$.

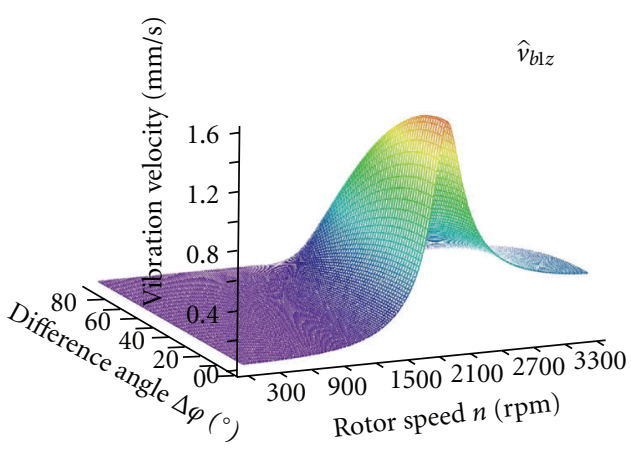

(a)

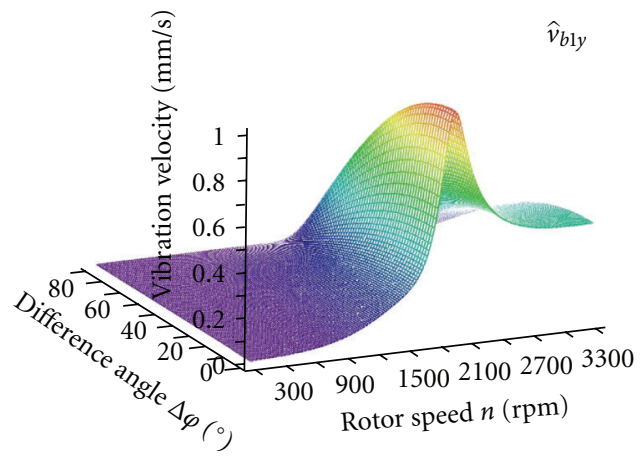

(c)

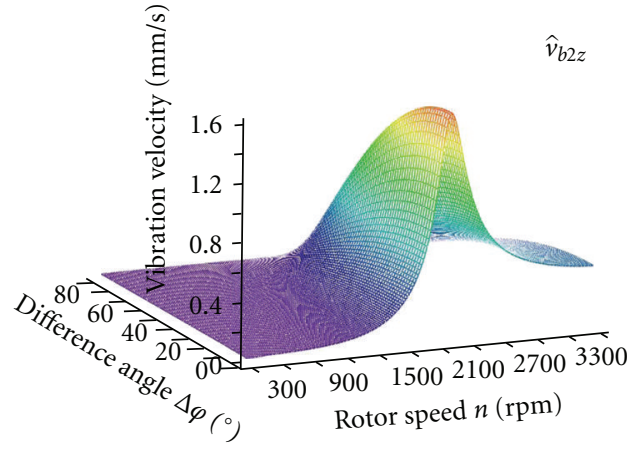

(b)

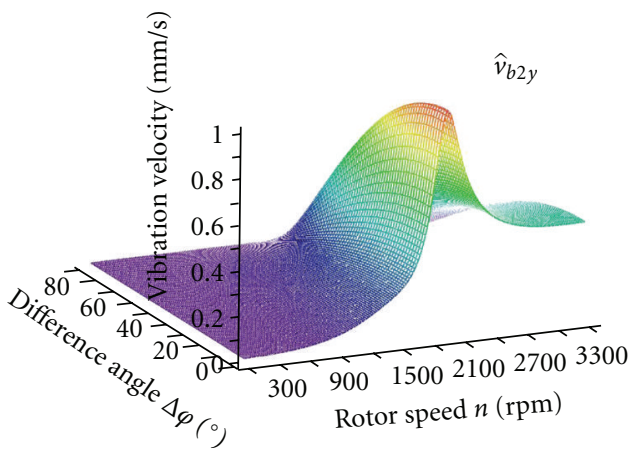

(d)

FIGURE 15: Bearing housing velocities in (a) vertical direction at bearing (1), (b) vertical direction at bearing (2), (c) horizontal direction at bearing (1), and (d) horizontal direction at bearing (2), depending on the rotor speed $n$ and on the difference angle $\Delta \varphi$. 
motor was shown. The presented simplified rotordynamic model can also be applied to rotating machines, other than electrical machines. In this case, only the electromagnetic spring value $c_{m}$ has to be put to zero.

\section{References}

[1] M. I. Friswell, J. E. T. Penny, S. D. Garvey, and A. W. Lees, Dynamics of Rotating Machines, Cambridge University Press, Cambridge, UK, 2010.

[2] L. Maurice and J. R. Adams, Rotating Machinery Vibration, CRC Press, Taylor \& Francis Group, Boca Raton, Fla, USA, 2010.

[3] J. M. Vance, F. J. Zeidan, and B. Murphy, Machinery Vibration and Rotordynamics, John Wiley \& Sons, Hoboken, NJ, USA, 2010.

[4] J. S. Rao, Rotor Dynamics, John Wiley \& Sons, New York, NY, USA, 1996.

[5] R. Gasch, R. Nordmann, and H. Pfützner, Rotordynamik, Springer, Berlin, Germany, 2002.

[6] W. Kliem, C. Pommer, and J. Stoustrup, "Stability of rotor systems: a complex modelling approach," Zeitschrift fur Angewandte Mathematik und Physik, vol. 49, no. 4, pp. 644-655, 1998.

[7] D. G. Dorrell, "Experimental behaviour of unbalanced magnetic pull in 3-phase induction motors with eccentric rotors and the relationship with tooth saturation," IEEE Transactions on Energy Conversion, vol. 14, no. 3, pp. 304-309, 1999.

[8] A. C. Smith and D. G. Dorrell, "Calculation and measurement of unbalanced magnetic pull in cage induction motors with eccentric rotors. I. Analytical model," IEE Proceedings: Electric Power Applications, vol. 143, no. 3, pp. 193-201, 1996.

[9] W. Schuisky, "Magnetic pull in electrical machines due to the eccentricity of the rotor," Electricity Research Association Transaction, vol. 295, pp. 391-399, 1972.

[10] T. P. Holopainen, Electromechanical interaction in rotor $d y$ namics of cage induction motors, Ph.D. thesis, VTT Technical Research Centre of Finland, Helsinki University of Technology, Finland, 2004.

[11] A. Arkkio, M. Antila, K. Pokki, A. Simon, and E. Lantto, "Electromagnetic force on a whirling cage rotor," IEE Proceedings: Electric Power Applications, vol. 147, no. 5, pp. 353-360, 2000.

[12] R. Belmans, A. Vandenput, and W. Geysen, "Calculation of the flux density and the unbalanced pull in two pole induction machines," Archiv für Elektrotechnik, vol. 70, no. 3, pp. 151$161,1987$.

[13] R. L. Stoll, "Simple computational model for calculating the unbalanced magnetic pull on a two-pole turbogenerator rotor due to eccentricity," IEE Proceedings: Electric Power Applications, vol. 144, no. 4, pp. 263-270, 1997.

[14] U. Werner, Rotordynamische analyze von asynchronmaschinen mit magnetischen unsymmetrien, dissertation, Technical University of Darmstadt, Shaker, Aachen, Germany, 2006.

[15] U. Werner, "Mathematical analysis of rotor shaft displacements in asynchronous machines; a critical speed or just a rotation of the orbit axis?" ZAMM Zeitschrift fur Angewandte Mathematik und Mechanik, vol. 89, no. 7, pp. 514-535, 2009.

[16] IEC 60034-14, Rotating electrical machines-part 14: mechanical vibration of certain machines with shaft heights $56 \mathrm{~mm}$ and higher-measurement, evaluation and limits of vibration severity, International Electrotechnical Commissio, 2007.
[17] ANSI /API 541, Form-wound-Squirrel-Cage Induction Motors-500 Horse power and Larger, API, 2004.

[18] O. Reynolds, "On the theory of lubrication," Philosophical Transaction of the Royal Society, vol. 177, pp. 157-234, 1886.

[19] A. A. Gnanadoss and M. R. Osborne, "The numerical solution of reynolds' equation for a journal bearing," Quarterly Journal of Mechanics and Applied Mathematics, vol. 17, no. 2, pp. 241246, 1964.

[20] J. Glienicke, Feder- und dämpfungskonstanten von gleitlagern für turbomaschinen und deren einfluss auf das schwingungsverhalten eines einfachen rotors, dissertation, Technische Hochschule Karlsruhe, 1966.

[21] J. Lund and K. Thomsen, "A calculation method and data for the dynamics of oil lubricated journal bearings," in Topics in Fluid Film Bearing and Rotor Bearing System Design and Optimization, pp. 1-28, ASME, New York, NY, USA, 1978.

[22] A. Tondl, Some Problems of Rotor Dynamics, Chapman \& Hall, London, UK, 1965. 

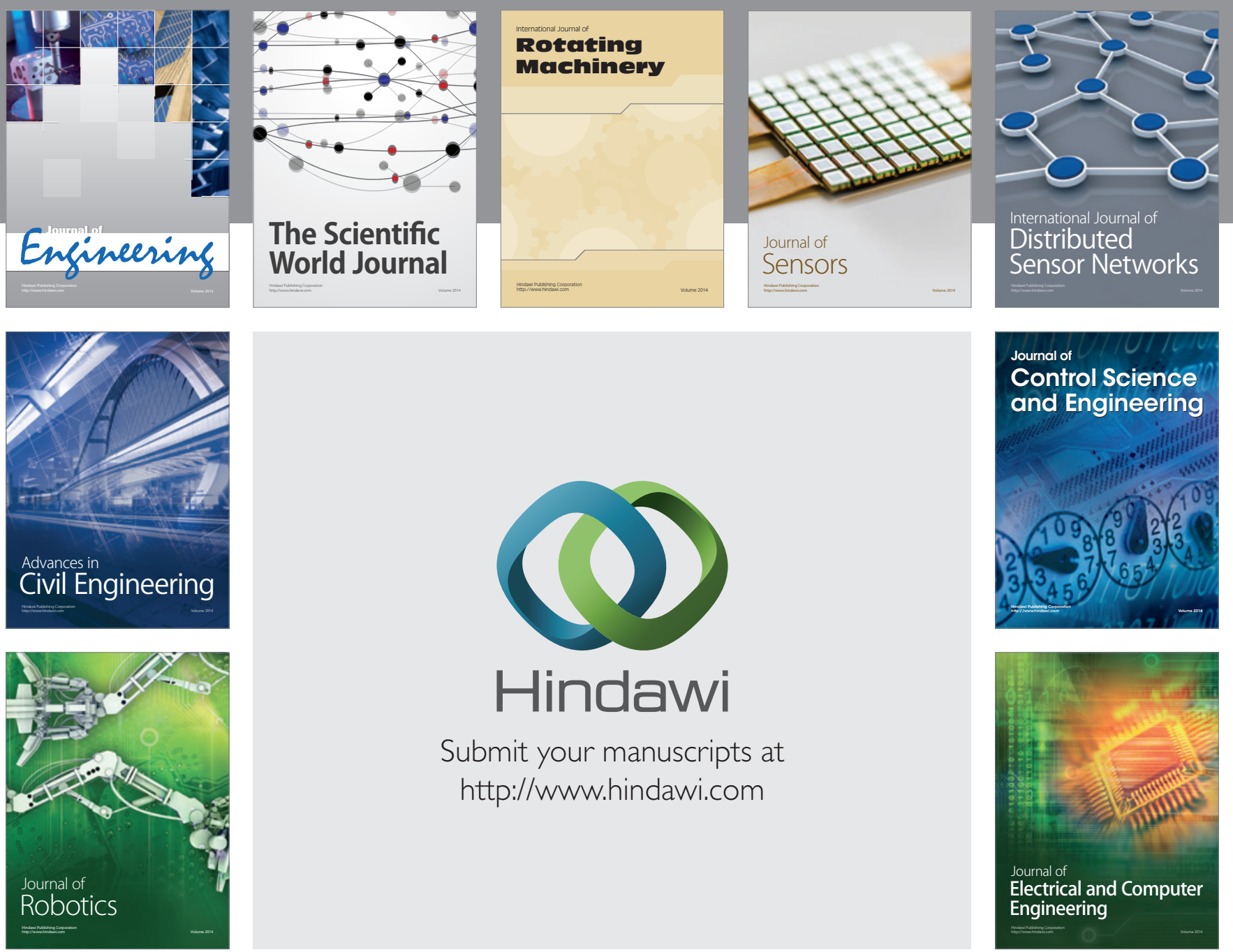

Submit your manuscripts at

http://www.hindawi.com
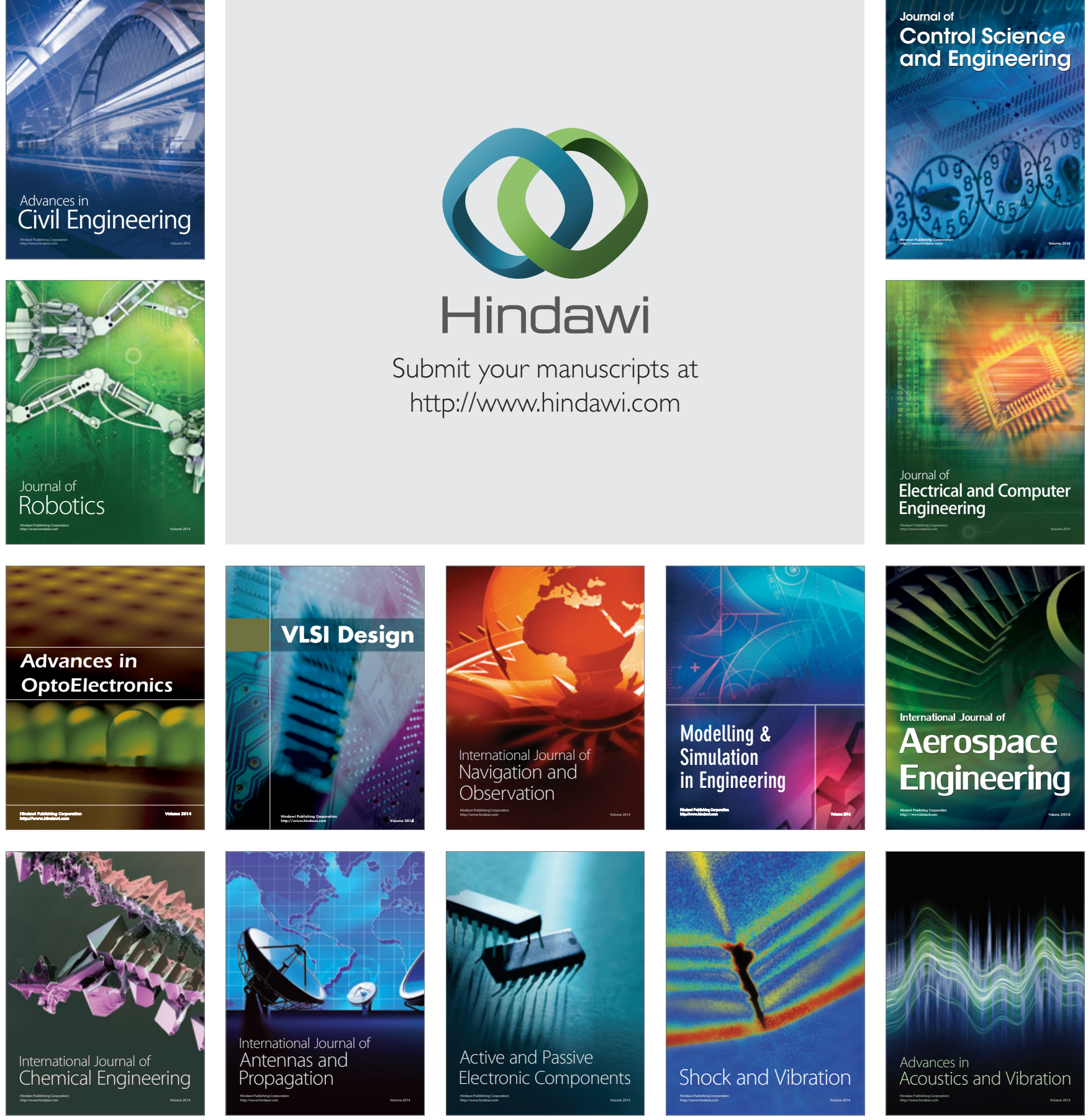